\title{
A New Formulation of Classical Mechanics-Part 2
}

\author{
Federico Petrovich \\ Departamento de Fisica, Facultad de Ciencias Exactas y Naturales, Universidad de Buenos Aires, Ciudad \\ Universitaria, Buenos Aires, Argentina \\ Email: fedepetrov@df.uba.ar
}

Received 18 March 2016; accepted 27 May 2016; published 30 May 2016

Copyright (C) 2016 by author and Scientific Research Publishing Inc.

This work is licensed under the Creative Commons Attribution International License (CC BY).

http://creativecommons.org/licenses/by/4.0/

(c) (i) Open Access

\begin{abstract}
In the first part of this paper, we found a more convenient algorithm for solving the equation of motion of a system of $\boldsymbol{n}$ bodies. This algorithm consists in solving first the trajectory equation and then the temporal equation. In this occasion, we will introduce a new way to solve the temporal equation by curving the horizontal axis (the time axis). In this way, we will be able to see the period of some periodic systems as the length of a certain curve and this will allow us to approximate the period in a different way. We will also be able to solve some problems like the pendulum one without using elliptic integrals. Finally, we will solve Kepler's problem using all the formalism.
\end{abstract}

\section{Keywords}

Classical Mechanics, Pendulum, Kepler's Problem

\section{Introduction}

We shall use the following notation given at the first part [1].

$/ / /$

Notation: Along this paper, we shall consider the variables $t$ and $\tilde{t}$. The derivatives respect to the variable $t$ will be denoted by the symbol "•" while the derivatives respect the variable $\tilde{t}$ will be denoted by the symbol apostrophe "'”. In addition, if $A_{i j} \in l R \forall 1 \leq i \leq n, 1 \leq j \leq 3$ we will denote:

- $A_{i} \equiv A_{i 1}+A_{i 2}+A_{i 3}$

- $\quad \bar{A}_{i} \equiv\left(A_{i 1}, A_{i 2}, A_{i 3}\right)$ 
- $A \equiv \sum_{i=1}^{n} A_{i} \equiv \sum_{i j} A_{i j}$

- $\bar{A} \equiv\left(A_{1}, \cdots, A_{n}\right)$

- $\quad \overline{\bar{A}} \equiv\left(\begin{array}{ccc}A_{11} & A_{12} & A_{13} \\ \vdots & \vdots & \vdots \\ A_{n 1} & A_{n 2} & A_{n 3}\end{array}\right)$.

III

In the first part of this paper, we study the problem of $n$ bodies interacting in a certain medium, which we will assume that it is the vacuum in this part (since the general case is analogous), whose equation of motion (in the vacuum case) is given by

$$
\left\{\begin{array}{l}
m_{i} \ddot{\bar{x}}_{i}(t)=\bar{F}_{i}(\overline{\bar{x}}(t)) \\
\overline{\bar{x}}\left(t_{0}\right)=\overline{\bar{x}}_{0} \quad \forall t \in I \\
\dot{\overline{\bar{x}}}\left(t_{0}\right)=\dot{\overline{\bar{x}}}_{0}
\end{array} \quad \forall t\right.
$$

where $m_{i}$ and $x_{i j}$ are the mass and the component $j$ of the position of the $i$-body respectively while $\bar{F}_{i}$ is the force applied to the $i$-body.

We saw that if $\tilde{\overline{\bar{X}}}: J \rightarrow l R^{3 \times n}$ is such that $\overline{\bar{X}}(t)=\tilde{\overline{\bar{X}}}(\tilde{t}(t))$ with

$$
\left\{\begin{array}{l}
\dot{\tilde{t}}(t)=u(\tilde{t}(t)) \\
\tilde{t}\left(t_{0}\right)=\tilde{t}_{0}
\end{array}\right.
$$

then Equation (1) (without the initial conditions) is equivalent to

$$
\left\{\begin{array}{l}
F_{i j}(\tilde{\bar{x}}(\tilde{t}))=u^{2}(\tilde{t}) m_{i} \tilde{x}_{i j}^{\prime}(\tilde{t}) \text { if } \tilde{x}_{i j}^{\prime}(\tilde{t})=0 \\
T_{i j}\left(t_{0}\right)+\tilde{W}_{i j}(\tilde{t})=u^{2}(\tilde{t}) \tilde{T}_{i j}(\tilde{t}) \text { if } \tilde{x}_{i j}^{\prime}(\tilde{t}) \neq 0
\end{array}\right.
$$

where

$$
\begin{gathered}
T_{i j}(t)=\frac{1}{2} m_{i} \dot{x}_{i j}^{2}(t) \\
\tilde{T}_{i j}(\tilde{t})=\frac{1}{2} m_{i} \tilde{x}_{i j}^{\prime 2}(\tilde{t}) \\
\tilde{W}_{i j}(\tilde{t})=\int_{\tilde{t}_{0}}^{\tilde{t}} F_{i j}(\tilde{\overline{\bar{X}}}(s)) \tilde{x}_{i j}^{\prime}(s) \mathrm{d} s .
\end{gathered}
$$

At the same time, we saw that Equation (3) and the initial conditions (and hence Equation (1)) are equivalent to

$$
\begin{gathered}
\left\{\begin{array}{l}
\tilde{\overline{\bar{W}}}(\tilde{t})+\overline{\bar{T}}\left(t_{0}\right) / /^{+} \tilde{\overline{\bar{T}}}(\tilde{t}) \\
\overline{\bar{x}}_{0}=\tilde{\overline{\bar{x}}}\left(\tilde{t}_{0}\right) \\
\overline{\overline{\bar{X}}}_{0} / / \tilde{\overline{\bar{x}}}^{\prime}\left(\tilde{t}_{0}\right)
\end{array} \quad \forall \tilde{t} \in J\right. \\
\tilde{x}_{i j}^{\prime}(\tilde{t})=\tilde{x}_{i j}^{\prime \prime}(\tilde{t})=0 \Rightarrow F_{i j}(\tilde{\overline{\bar{x}}}(\tilde{t}))=0 \\
\tilde{\overline{\bar{x}}}^{\prime}(\tilde{t})=0 \Rightarrow \overline{\bar{F}}(\tilde{\overline{\bar{x}}}(\tilde{t})) / /^{+}\left(\begin{array}{cc}
m_{1} & \tilde{\bar{x}}^{\prime}(\tilde{t}) \\
m_{n} & \tilde{\bar{x}}_{n}^{\prime \prime}(\tilde{t})
\end{array}\right) \forall \tilde{t} \in J
\end{gathered}
$$




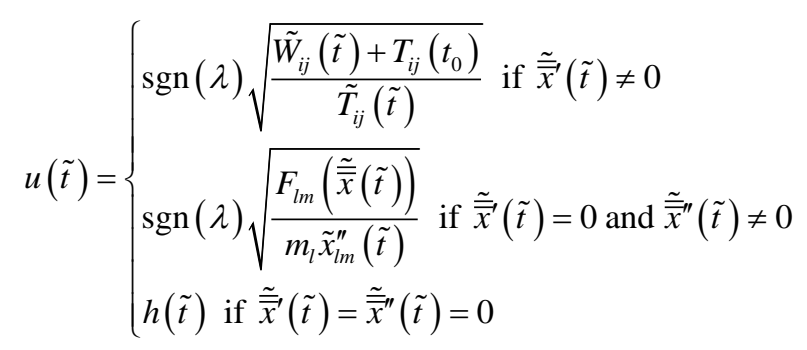

where $h$ can be any function, $i, j, l$ and $m$ are arbitrary indexes satisfying $\tilde{x}_{i j}^{\prime}(\tilde{t}) \neq 0, \tilde{x}_{l m}^{\prime \prime}(\tilde{t}) \neq 0$ and $\lambda$ is given by

$$
\overline{\bar{x}}_{0}=\lambda \tilde{\overline{\bar{x}}}^{\prime}\left(\tilde{t}_{0}\right) .
$$

We also proved that if $\tilde{\overline{\bar{X}}}$ is a parametrization of $C$, where $C$ is the image of $\overline{\bar{x}}$, then it satisfies conditions (7), (8) and (9). Due to this fact, we call trajectory equation to condition (7), since $C$ represents the trajectory of the system and conditions (8), (9) are just extra conditions for particular cases. We finally saw that if $\tilde{\overline{\bar{X}}}^{*}: J^{*} \rightarrow I R^{3 \times n}$ satisfies conditions (7), (8) and (9), then $\tilde{\overline{\overline{\bar{X}}}}: J \rightarrow I R^{3 n}$ given by $\tilde{\overline{\bar{x}}}(\tilde{t})=\tilde{\overline{\bar{X}}}^{*}(\tau(\tilde{t}))$ also satisfies them, with $\tau: J \rightarrow J^{*}$ such that $\tau\left(\tilde{t}_{0}\right)=\tilde{t}_{0}$ and $\tau^{\prime}(\tilde{t})>0$. In addition

$$
u(\tilde{t})=\frac{u^{*}(\tau(\tilde{t}))}{\tau^{\prime}(\tilde{t})} .
$$

On the other hand, we call temporal equation to Equation (2), since it determines the relationship between $t$ and $\tilde{t}$. We also call temporal equation to Equation (10). We proved that if the force comes from a potential $V$, then we can write Equation (10) (for $\tilde{\overline{\bar{x}}}(\tilde{t}) \neq 0$ ) using the mechanical energy of the system as follows

$$
u(\tilde{t})=\operatorname{sgn}(\lambda) \sqrt{\frac{E-V(\tilde{\bar{X}}(\tilde{t}))}{\tilde{T}(\tilde{t})}}
$$

where $E$ is the energy.

Based on these results, we develop the following algorithm for solving Equation (1):

1) Find a solution $\tilde{\overline{\bar{X}}}^{*}: J^{*} \rightarrow I R^{3 \times n}$ of the trajectory equation and check that it satisfies conditions (8) and (9).

2) Choose conveniently a function $\tau: J \rightarrow J^{*}$ with $\tau\left(\tilde{t}_{0}\right)=\tilde{t}_{0}$ and $\tau^{\prime}(\tilde{t})>0$ in order to build another solution $\tilde{\overline{\bar{x}}}: J \rightarrow I R^{3 \times n}$ given by $\tilde{\overline{\bar{x}}}(\tilde{t})=\dot{\overline{\bar{x}}}^{*}(\tau(\tilde{t}))$.

3) Find the function $u$ given in Equation (10) (or (13)).

4) Solve the temporal equation.

Finally, $\overline{\bar{x}}(t)=\tilde{\bar{x}}(\tilde{t}(t))$ is the solution of Equation (1).

We saw that if we want to solve the equation of motion, it is more convenient to follow this algorithm.

In this occasion, we will change the last step of this algorithm, i.e., we will find a new way to solve the temporal equation, by curving the horizontal axis (the time axis). In this way we will be able to solve some problems that cannot be solved in the traditional way (without using elliptic integrals) like the pendulum problem. We will also be able to see the period of some periodic systems as the length of a curve. This fact can be compared with the Hamilton-Lagrange's formalism, where we can see the period of some systems as the area of a surface given by the phase space [2].

First, we will use the arc length function and its inverse in order to introduce a new way to graph functions by curving the axes. Then, we will use these results in order to complete the algorithm and to graph each component of the solution of Equation (1) in this way. Finally, we will solve the harmonic oscillator, the pendulum, the particle under the action of two elastic springs [3] and Kepler's problems using all the formalism.

\section{A New Way to Graph Functions by Curving the Axes}

In this section we will use the arc length function and its inverse in order to introduce a new way to graph functions by curving the axes. Before doing this, we will introduce the notation that will be used and we will give 
some definitions.

III

Notation and preliminaries: let $C$ be a curve in the plane given by a parametrization $\gamma:[a, b] \subseteq l R \rightarrow C$ with $\gamma(t)=(x(t), y(t))$.

- We will denote by $J_{x}=\{x \in l R:(x, y) \in C\}$ and $J_{y}=\{y \in l R:(x, y) \in C\}$, i.e., $J_{x}$ is the image of $x(t)$ and $J_{y}$ is the image of $y(t)$.

- We will assume that $0 \in[a, b]$ and we will denote by $P_{0}=\left(x_{0}, y_{0}\right)=\gamma(0)$.

- If $l(a)$ and $l(b)$ are the lengths of the curve from $P_{0}$ to $\gamma(a)$ and $\gamma(b)$ respectively, we write $L=(-l(a), l(b))$.

Definition 1: let $C$ be a curve in the plane describing the graph of a function depending of $x$ and given by a parametrization $\gamma:[a, b] \rightarrow C$. We define the function $l_{\gamma}[x]: J_{x} \rightarrow L$ as follows: $l_{\gamma}[x](x)=s$ if and only if $\mathrm{s}$ is either $+l$ or $-l$, where $l$ is the length of the curve $C$ measured from $P_{0}$ to $(x, y)$ and the sign depends of the orientation of the parametrization as shown in Figure 1 and Figure 2.

In a similar way, if $C$ describes the graph of a function depending of $y$, we define the function $l_{\gamma}[y]: J_{y} \rightarrow L$ as shown in Figure 3 and Figure 4.

Definition 2: let $C$ be a curve in the plane given by a parametrization $\gamma:[a, b] \rightarrow C$. We define the function $x_{\gamma}: L \rightarrow J_{x}$ as follows: $x_{\gamma}(s)=x$ if and only if $\mathrm{s}$ is either $+l$ or $-l$, where $l$ is the length of the curve $C$ measured from $P_{0}$ to $(x, y)$ and the sign depends of the orientation of the parametrization as shown in Figure 5 and Figure 6.

In a similar way, we define the function $y_{\gamma}: L \rightarrow J_{y}$ as shown in Figure 7 and Figure 8.

III

Before proceeding to the new way to graph functions, we will discuss some points about these functions.

On the one hand, note that all these functions are uniquely determined by the curve $C$ described implicitly by $F(x, y)=0$, the initial point $P_{0}$ and the orientation. In addition, there are infinite parametrizations that define the same function. Then, in the examples that we will see later, we will find $F, P_{0}$ and the orientation in order to determine these functions, i.e., we will not find the parametrization $\gamma$.

On the other hand, if $C$ describes the graph of a function $y: J_{x} \rightarrow J_{y}$ depending of $x$, by Definition 1 it is

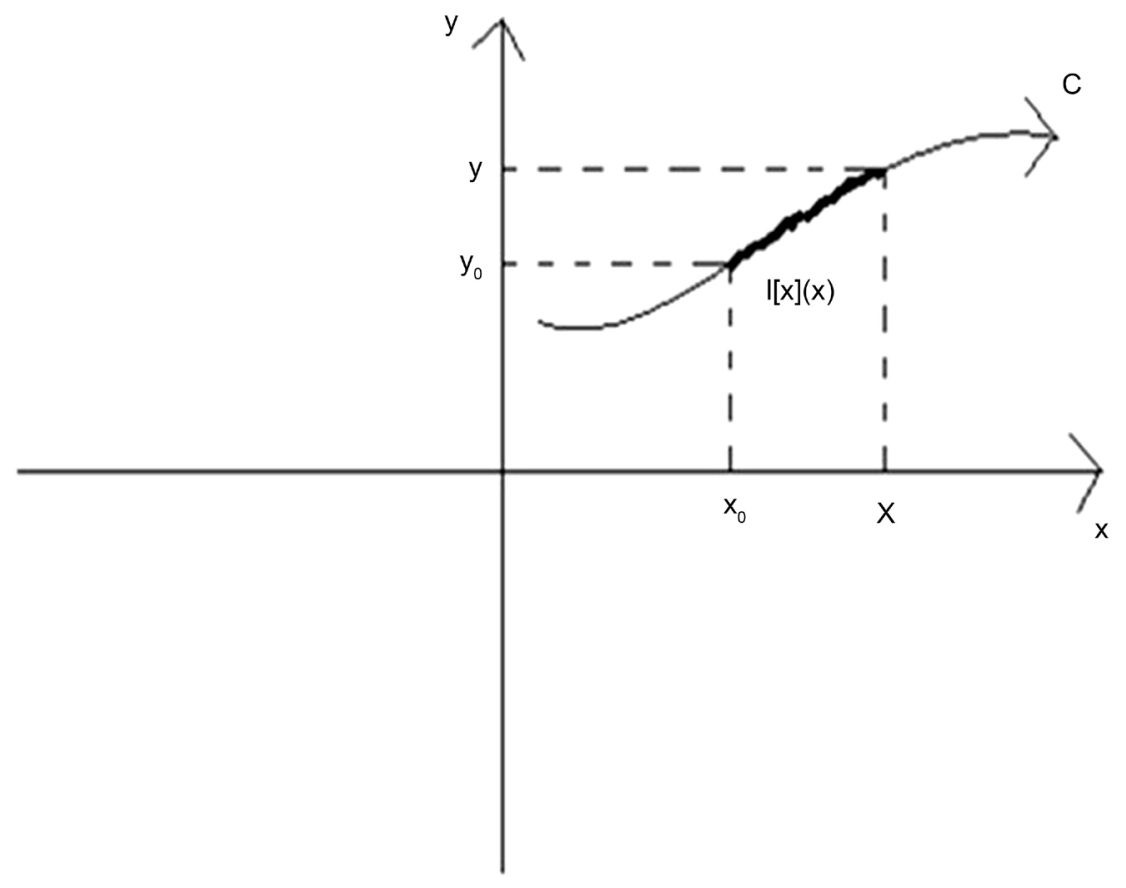

Figure 1. In this case $l_{\gamma}(x)$ is positive since its sense coincides with the orientation. 


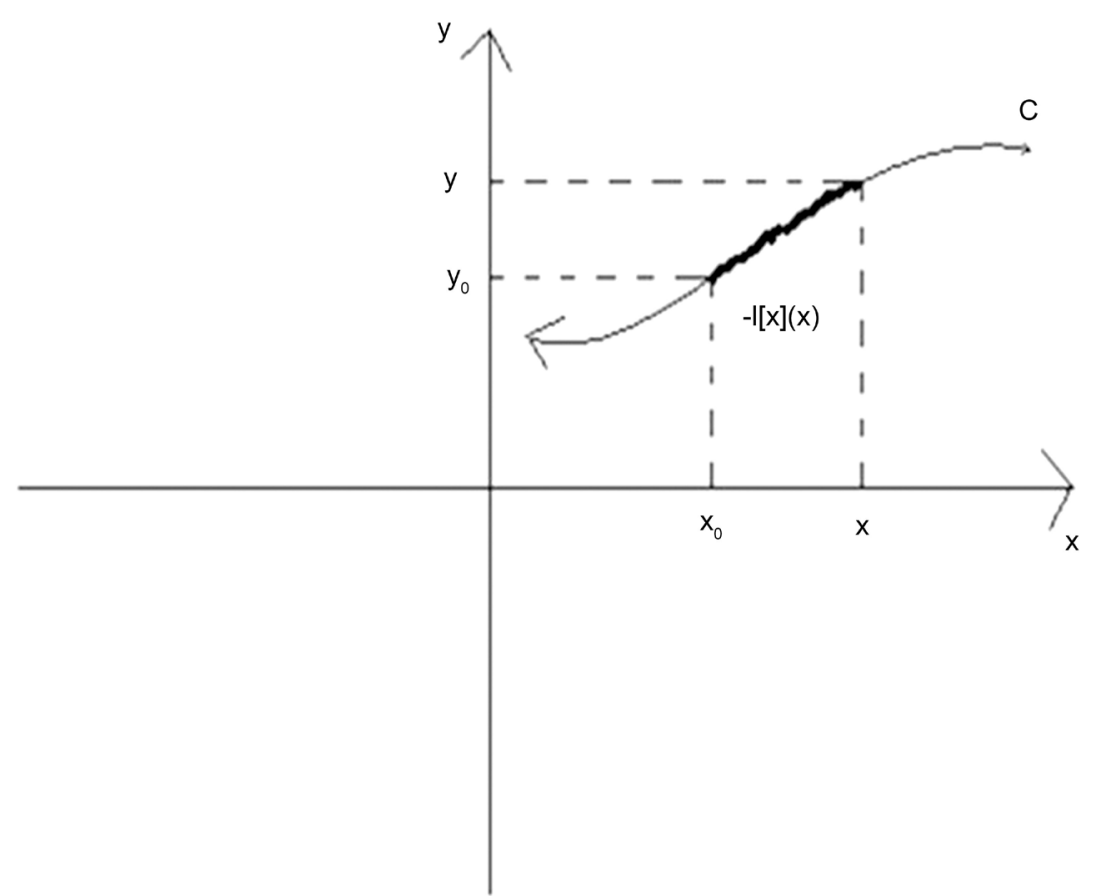

Figure 2. In this case $l_{\gamma}(x)$ is negative since its sense is opposite to the orientation.

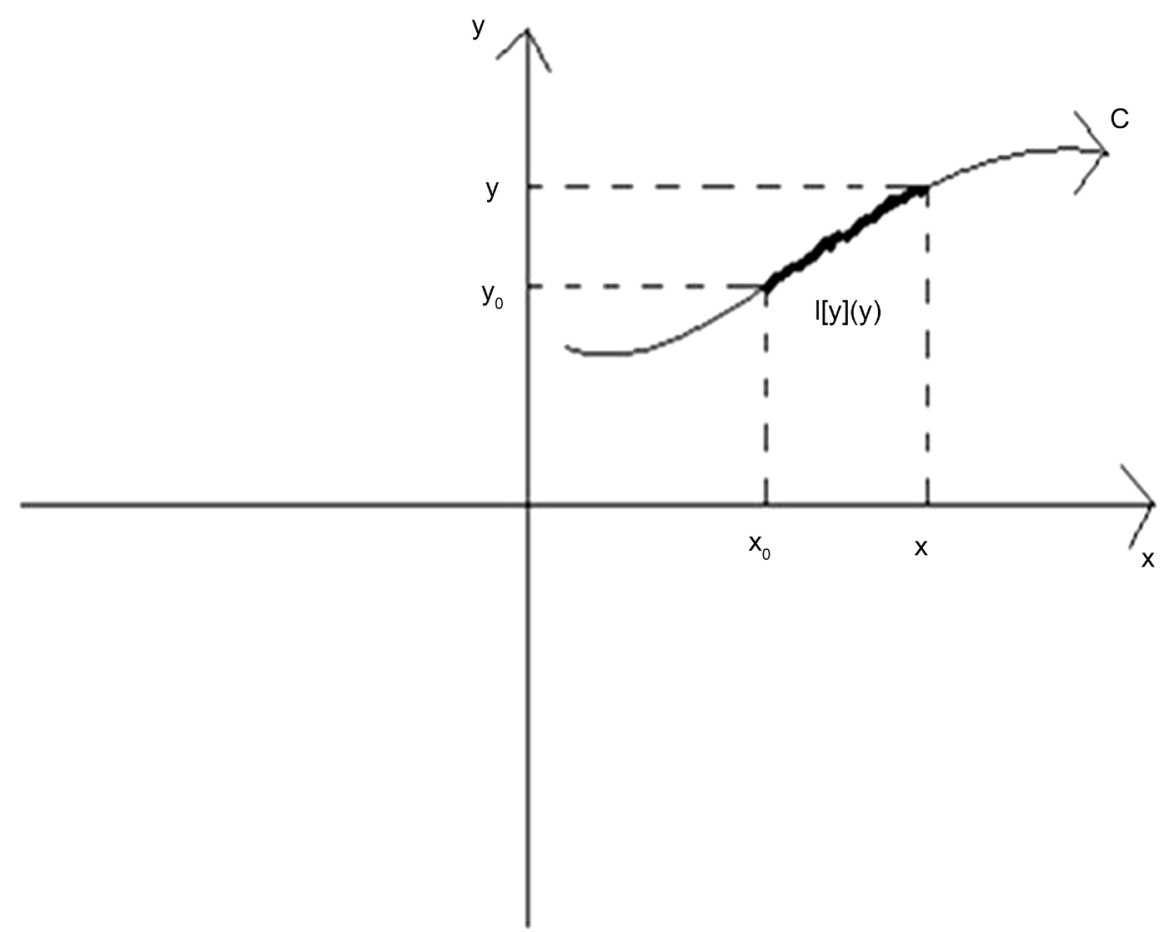

Figure 3. In this case $l_{\gamma}(x)$ is positive since its sense coincides with the orientation.

easily proved that $l_{\gamma}[x]$ is given by

$$
l_{\gamma}[x](x)= \pm \int_{x_{0}}^{x} \sqrt{1+\frac{\mathrm{d} y^{2}}{\mathrm{~d} x}(s)} \mathrm{d} s
$$






Figure 4. In this case $l_{\gamma}(y)$ is negative since its sense is opposite to the orientation.

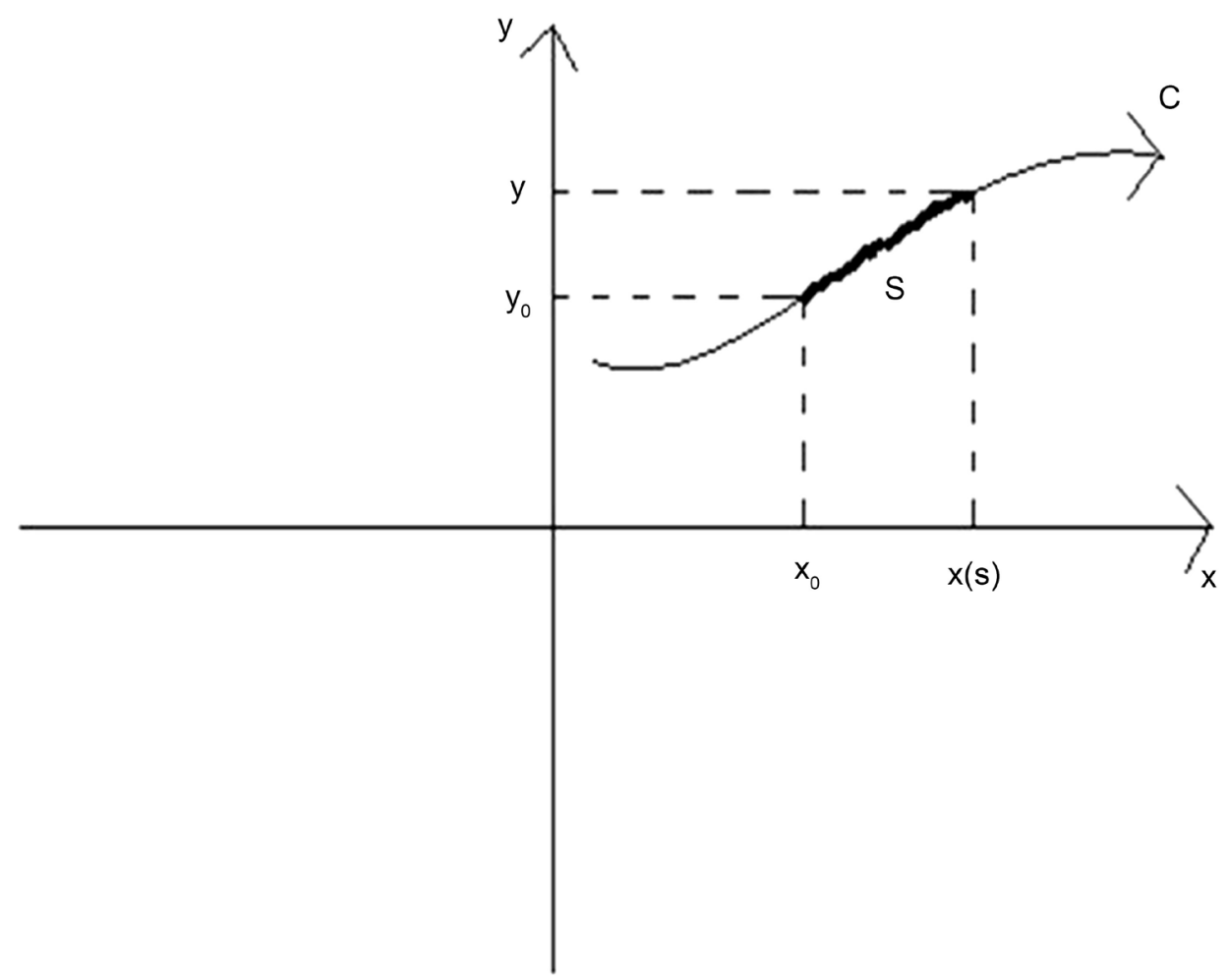

Figure 5. In this case $s=l$, i.e., $s$ coincides with length of the curve measured from $P_{0}$ to $(x, y)$ since it is in the same sense of the orientation. 


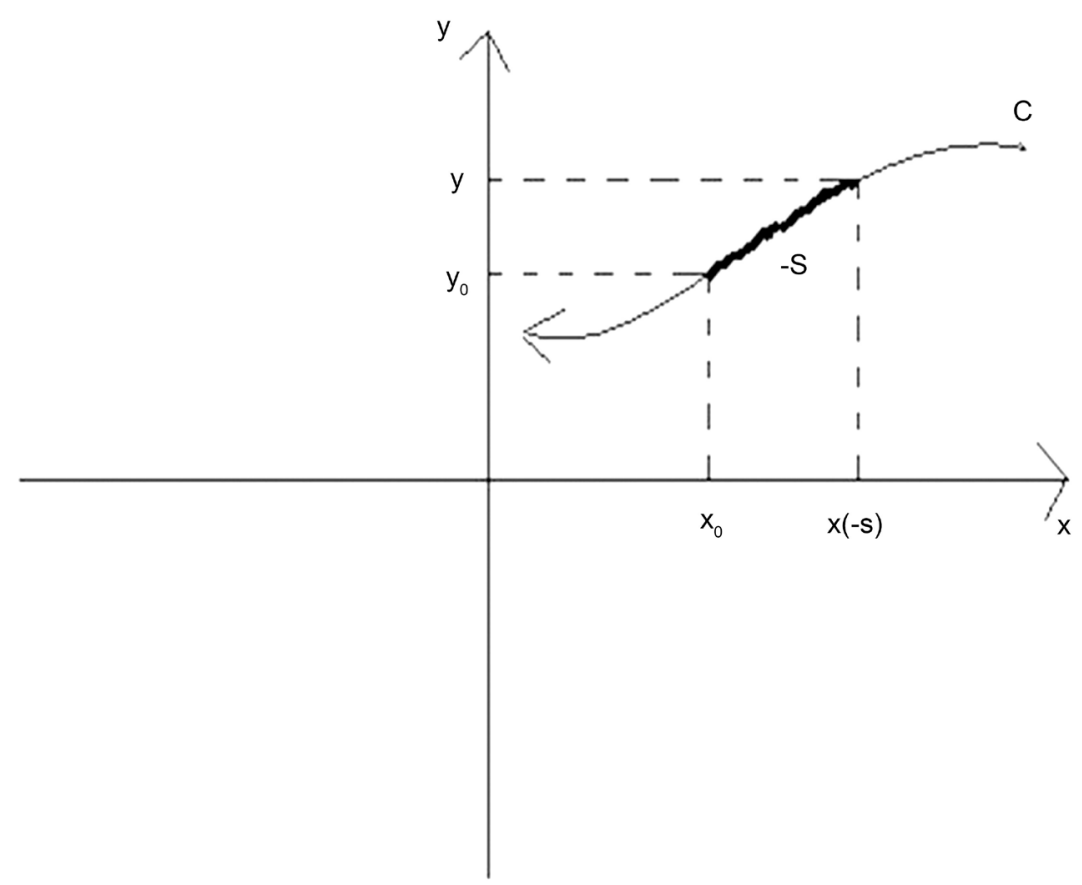

Figure 6. In this case $s=-l$, where $l$ is the length of the curve measured from $P_{0}$ to $(x, y)$, since its sense is opposite to the orientation.

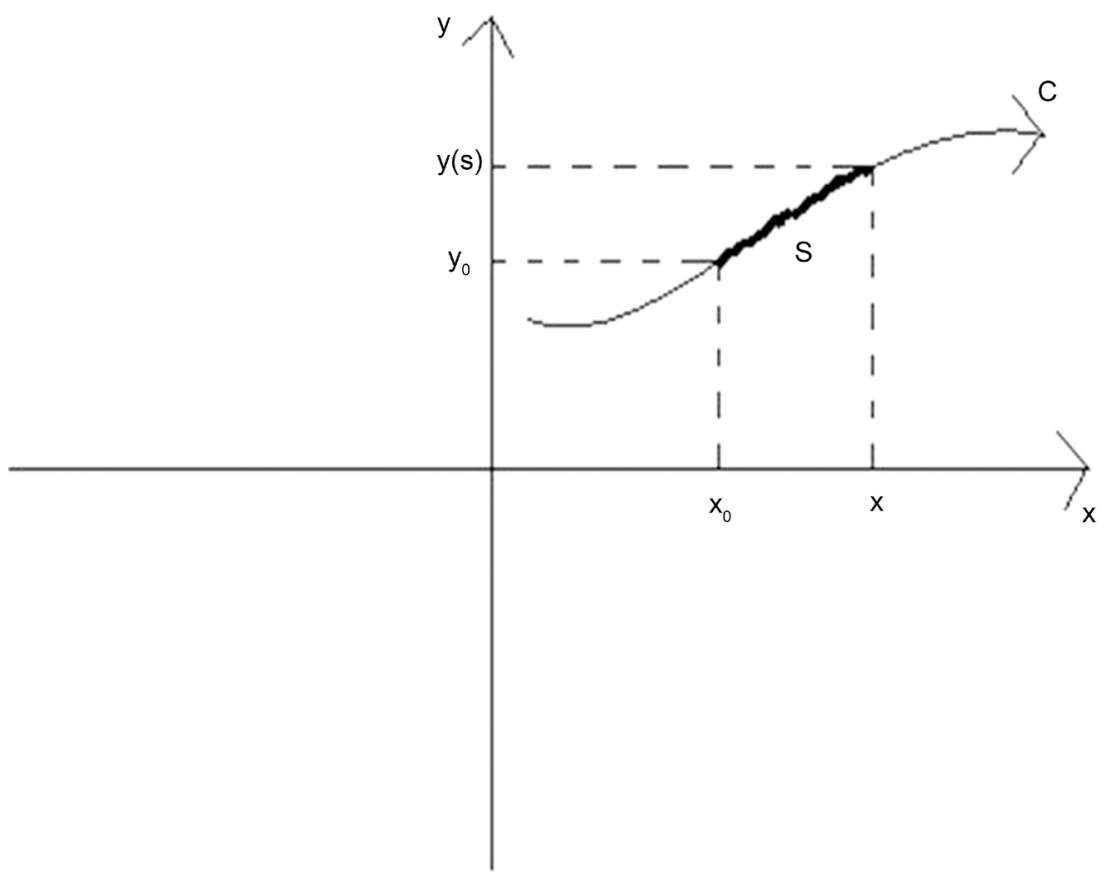

Figure 7. In this case $s=l$, i.e., $s$ coincides with length of the curve measured from $P_{0}$ to $(x, y)$ since it is in the same sense of the orientation.

where the sign + corresponds to the orientation given in Figure 1 and the sign - to the orientation given in Figure 2.

In addition, in this case we can see in Definition 2 that $x_{\gamma}$ is the inverse function of $l_{\gamma}[x]$ and then it follows from the inverse function theorem and Equation (14) that $x_{\gamma}$ is given by 


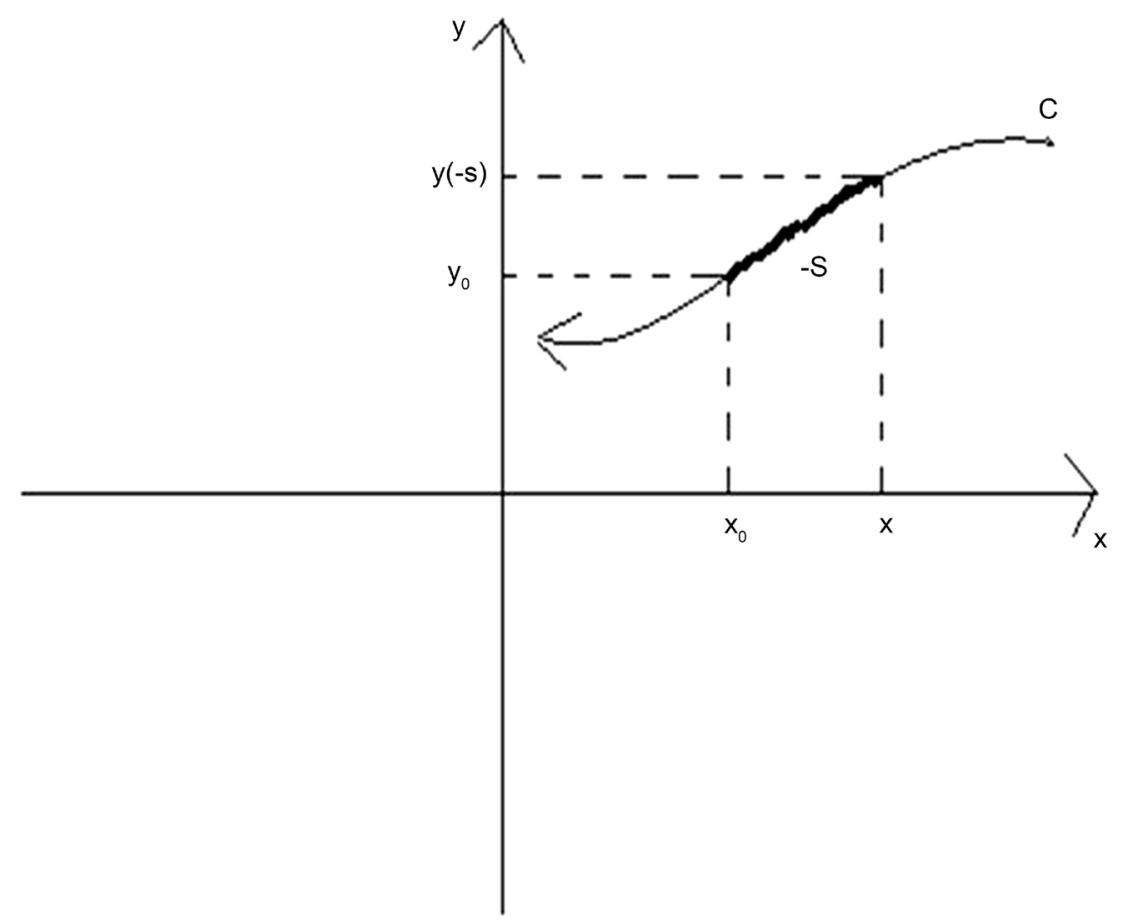

Figure 8. In this case $s=-l$, where $l$ is the length of the curve measured from $P_{0}$ to $(x, y)$, since its sense is opposite to the orientation.

$$
\left\{\begin{array}{l}
\frac{\mathrm{d} x_{\gamma}}{\mathrm{d} s}(s)= \pm \frac{1}{\sqrt{1+\frac{\mathrm{d} y^{2}}{\mathrm{~d} x}\left(x_{\gamma}(s)\right)}} \\
x_{\gamma}(0)=x_{0}
\end{array}\right.
$$

where the sign + corresponds to the orientation given in Figure 5 and the sign-to the orientation given in Figure 6.

If $C$ describes the graph of a function $x: J_{y} \rightarrow J_{x}$ depending of $y$ the process is analogous by changing $l_{\gamma}[x]$ and $x_{\gamma}$ by $l_{\gamma}[y]$ and $y_{\gamma}$.

Next, we will use these functions in order to graph functions by curving the axes.

//I

Curvilinear vertical axis: assume we have a differential equation whose solution is given by the function $X(t)=l_{\gamma}[y](\tilde{X}(t))$ where the curve $C$ is given by $x=x(y)$, it is oriented as in Figure 3 and $P_{0}=\left(x_{0}, y_{0}\right)$ (with $x_{0}=x\left(y_{0}\right)$ ). Then, we can graph this function as shown in Figure 9.

This way of making the graph of the function is equivalent to make the graph of the function $\tilde{X}$ by curving the $\mathrm{X}$-axis with the function $x$. Note that the origin of the horizontal axis is $(0,0)$ (i.e. it does not change) while the origin of the vertical axis is $P_{0}$. Note also that the original X-axis is not used to make the graph of the function, it is only used to perform the new $y$-axis and to make the graph of the function $\tilde{X}$. Finally, we can graph $X$ versus $t$ in a more simple way as shown in Figure 10.

If the curve $C$ is oriented as in Figure 4 the direction of the $\mathrm{X}$-axis changes.

Curvilinear horizontal axes: assume now that we have a differential equation whose solution is given by the function $X(t)=\tilde{X}\left(x_{\gamma}(t)\right)$ where the curve $C$ is given by $y=y(x)$, it is oriented as in Figure 5 and $P_{0}=\left(x_{0}, y_{0}\right)$ (with $y_{0}=y\left(x_{0}\right)$ ). Then, we can graph this function as shown in Figure 11.

This way of making the graph of the function is equivalent to make the graph of the function $\tilde{X}$ by curving the t-axis. Note that the origin of the vertical axis is $(0,0)$ (i.e. it does not change) while the origin of the horizontal axis is $P_{0}$. Note also that the original t-axis is not used to make the graph of the function, it is only used to perform the new t-axis and to make the graph of the function $\tilde{X}$. Like the previous case, we can graph 


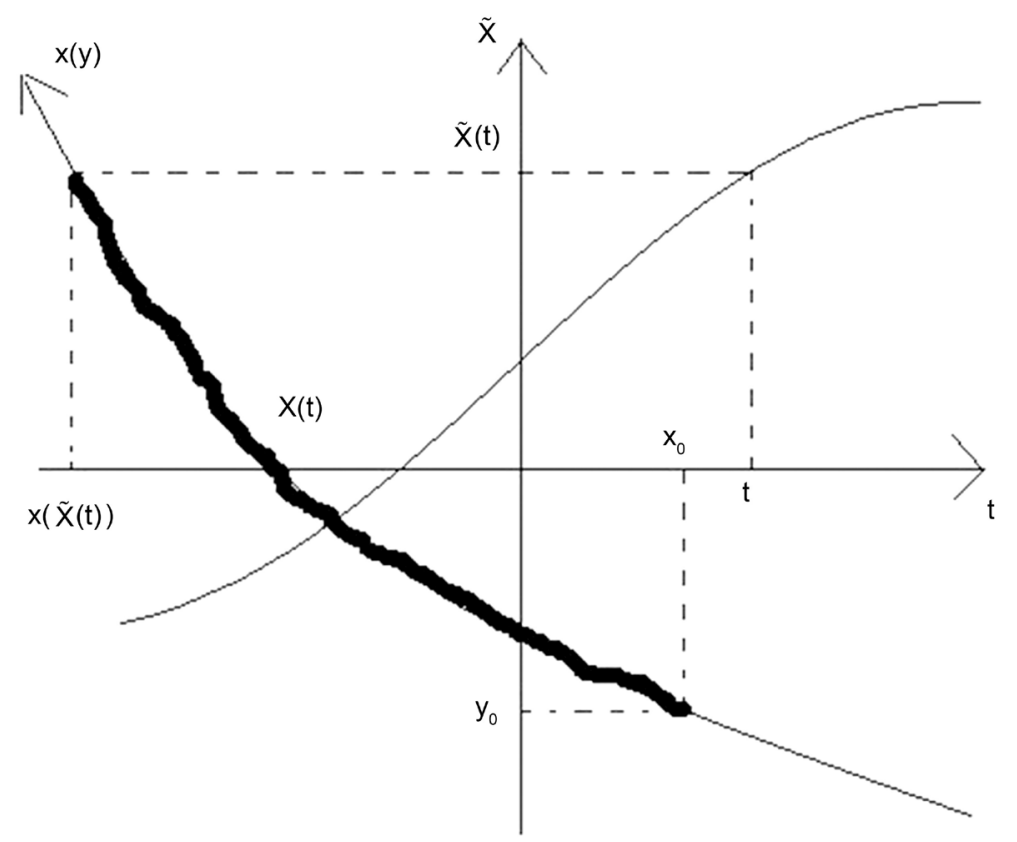

Figure 9. Graph of the function $X(t)=l_{\gamma}[y](\tilde{X}(t))$.

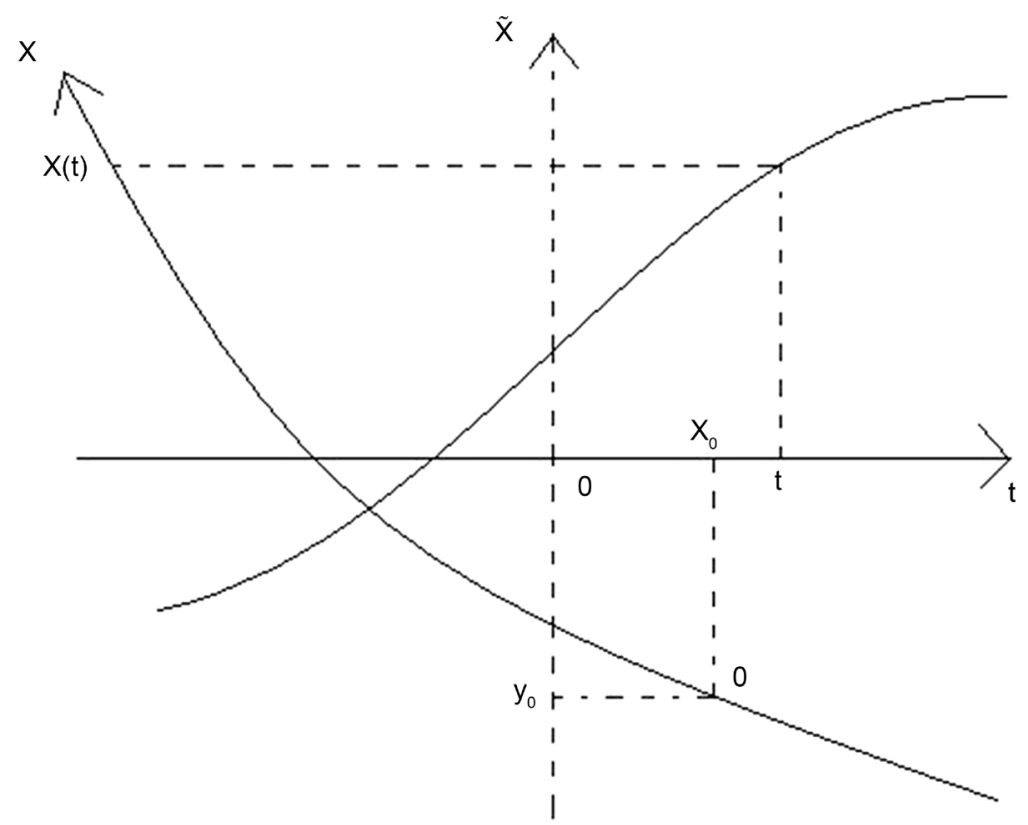

Figure 10. Graph of the function $X$ by curving the $\mathrm{X}$-axis.

$X$ versus $t$ in a more simple way as shown in Figure 12 .

If the curve $C$ is oriented as in Figure 6 the direction of the $\mathrm{X}$-axis changes.

If $C$ is not the graph of a function the idea is the same. For example if $C$ is a closed curve and it is positively oriented, the graph of $X$ versus $t$ is shown in Figure 13. In this case we need to say how many times the parametrization of the curve "turns". If it turns indefinitely, then $X:[0, \infty) \rightarrow I R$ and we can see in Figure 13 that $X$ is a periodic function whose period is given by the length of $C$.

$$
/ / /
$$

Next, we will complete the algorithm for solving the equation of motion by using these results. We will only 


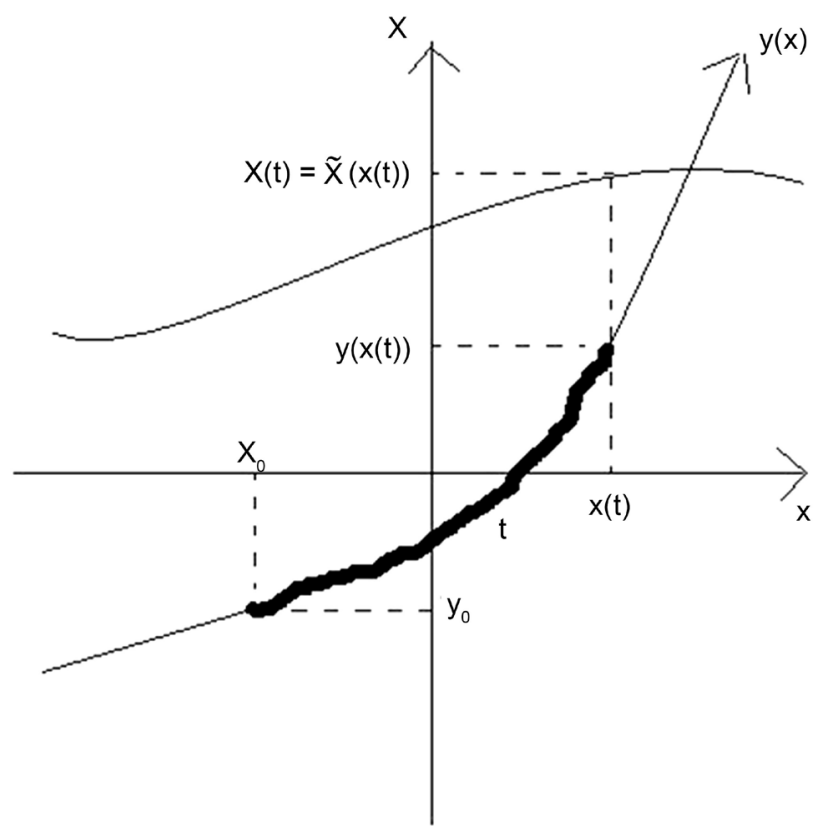

Figure 11. Graph of the function $X(t)=\tilde{X}\left(x_{\gamma}(t)\right)$.

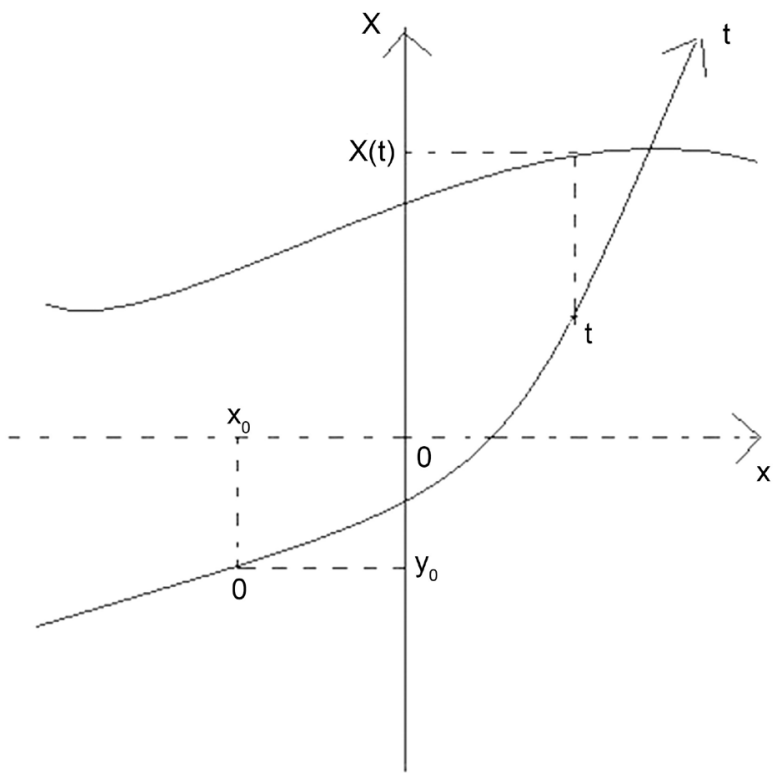

Figure 12. Graph of the function $X$ by curving the $t$-axis.

use the $x_{\gamma}$ functions, since we will just curve the t-axis.

\section{A New Way to Solve the Temporal Equation}

In the first part of this paper we find a more convenient algorithm for solving the equation of motion of a system of $n$ bodies. In this section we will use the $x_{\gamma}$ functions in order to change the step four of that algorithm, by solving the temporal equation in a different way.

Before doing that, we will need the following mathematical notion.

III

Definition 3: let $f: J \rightarrow I R$ and $x_{0} \in l R$. We define the following set $K\left(f, x_{0}\right)$ : 


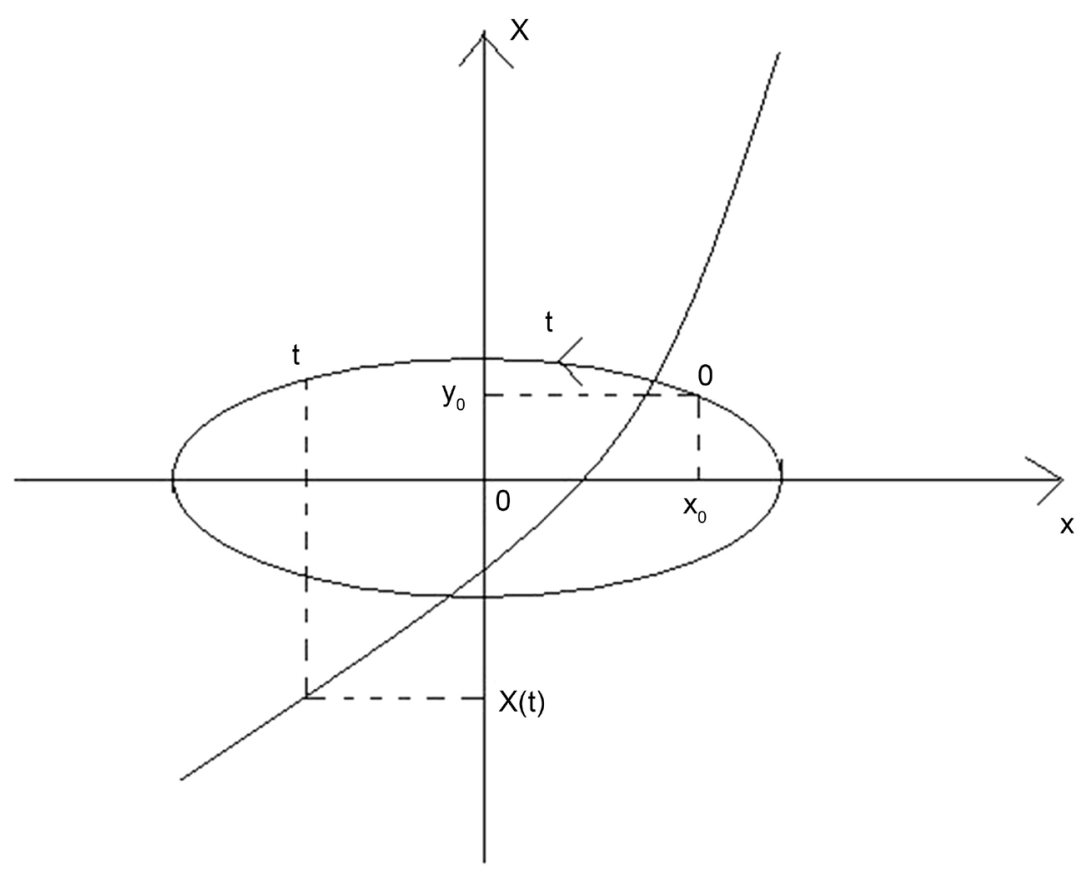

Figure 13. Graph of the function $X$ when the $t$-axis is a closed curve.

$$
K\left(f, x_{0}\right)=\left\{x \in J: \sqrt{\frac{1}{f^{2}}-1} \text { is integrable in }\left[x_{0}, x\right]\right\}
$$

Note 1: in order an element $x$ belongs to $K\left(f, x_{0}\right)$ it is necessary (but not sufficient) that in order the term which appears under the radical be no negative.

Note 2: if we do not choose the element $x_{0}$ appropriately (for instance if $\left|f\left(x_{0}\right)\right|>1$ ) then the set $K\left(f, x_{0}\right)$ can be empty.

Note 3: although $f(x)=0$, it may be possible that $x$ belongs to $K\left(f, x_{0}\right)$, since the improper integral could converge. Analogously, if $f\left(x_{0}\right)=0$, it may be possible that the set $K\left(f, x_{0}\right)$ is not empty for the same reason.

Proposition 1: let $x: I \rightarrow J$ and $u: J \rightarrow I R$ be such that

$$
\left\{\begin{array}{l}
\frac{\mathrm{d} x}{\mathrm{~d} s}(s)=u(x(s)) \\
x(0)=x_{0}
\end{array} .\right.
$$

Let also $y: K\left(u, x_{0}\right) \rightarrow I R$ be such that

$$
y(x)= \pm \int_{x_{0}}^{x} \sqrt{\frac{1}{u^{2}(s)}-1} \mathrm{~d} s+y_{0}
$$

where $y_{0} \in I R$ and the sign is arbitrary.

Then, if $u$ does not change the sign in the interval $K\left(u, x_{0}\right), x_{\gamma}: L \rightarrow K\left(u, x_{0}\right)$ is the solution of the differential equation where $C$ is described by $y=y(x), P_{0}=\left(x_{0}, y_{0}\right)$, and the curve is oriented as follows

- If $u$ is positive, then $C$ is oriented as in Figure 5 .

- $\quad$ If $u$ is negative, then $C$ is oriented as in Figure 6 .

Proof: by Equation (17) we have

$$
\frac{\mathrm{d} y}{\mathrm{~d} x}(x)= \pm \sqrt{\frac{1}{u^{2}(x)}-1}
$$


It follows that

$$
u(x)= \pm \frac{1}{\sqrt{1+\frac{\mathrm{d} y^{2}}{\mathrm{~d} x}(x)}} \forall x \in K\left(u, x_{0}\right)
$$

where the sign is + provided $u \geq 0$ and the sign is - provided $u \leq 0$.

Then, according to Equation (15) we have that $x(s)=x_{\gamma}(s)$ is the solution of Equation (16) where $P_{0}=\left(x_{0}, y_{0}\right)$ and $C$ is described by $y=y(x)$ with its respective orientation.

By Proposition 1, we will complete the algorithm for solving the equation of motion.

We will assume that the initial time $t_{0}=0$, which implies that Equation (2) is written as Equation (16) by taking $\tilde{t}_{0}=x_{0}$. In addition, we can see in Equation (10) (or (13)) that $u$ does not change its sign, and hence we can use Proposition 1. Then, we will change the algorithm as follows

1) Find a solution $\tilde{\overline{\bar{X}}}^{*}: J^{*} \rightarrow I R^{3 \times n}$ of the trajectory equation and check that it satisfies conditions (8) and (9).

2) Choose conveniently a function $\tau: J \rightarrow J^{*}$ with $\tau\left(\tilde{t}_{0}\right)=\tilde{t}_{0}$ and $\tau^{\prime}(\tilde{t})>0$ in order to build another solution $\tilde{\overline{\bar{X}}}: J \rightarrow l R^{3 \times n}$ given by $\tilde{\overline{\bar{X}}}(\tilde{t})=\tilde{\overline{\bar{X}}}^{*}(\tau(\tilde{t}))$.

3) Find the function $u$ given in Equation (10) (or (13)).

4) Find a function $y$ that satisfies Equation (17).

Finally, $\overline{\bar{x}}(t)=\tilde{\bar{x}}\left(x_{\gamma}(t)\right)$ is the solution of Equation (1) where $C$ is described by $y=y(x), \quad P_{0}=\left(x_{0}, y_{0}\right)$ and the curve is oriented as follows

- If $\lambda$ is positive, then $C$ is oriented as in Figure 5.

- If $\lambda$ is negative, then $C$ is oriented as in Figure 6.

where $\lambda$ is given by Equation (11).

In this way, we can graph each component of $\overline{\bar{X}}$ as in Figure 12 (or 13) by curving the horizontal axis, i.e., the time axis.

Note that we have only changed the step four. However, there is also a big difference in the step two.

In the step two of the old algorithm, the statement "choose conveniently a function $\tau: J \rightarrow J^{*}$ " refers to choose $\tau$ so that the temporal equation can be solved easily. However, according to Equation (12) we have

$$
\frac{\mathrm{d} \tilde{t}}{\mathrm{~d} t}=u(\tilde{t})=\frac{u^{*}(\tau(\tilde{t}))}{\tau^{\prime}(\tilde{t})} .
$$

This implies that

$$
\frac{\mathrm{d} \tilde{t}}{\mathrm{~d} t} \frac{\mathrm{d} \tau}{\mathrm{d} \tilde{t}}=u^{*}(\tau(\tilde{t}))
$$

Hence we arrive to

$$
\frac{\mathrm{d} \tau}{\mathrm{d} t}=u^{*}(\tau)
$$

Then, we can see that choosing $\tau$ so that the temporal equation can be solved easily is equivalent to make a simple change of variables in the temporal equation. This implies that if the solution of $\frac{\mathrm{d} \tilde{t}}{\mathrm{~d} t}=u^{*}(\tilde{t})$ is a non-elemental function, then the solution of $\frac{\mathrm{d} \tilde{t}}{\mathrm{~d} t}=u(\tilde{t})$ will also be a non-elemental function. Hence, we can say that in the old algorithm, the step two is not very important.

In the step two of the new algorithm, the statement "choose conveniently a function $\tau: J \rightarrow J^{*}$ " refers to choose $\tau$ so that $\left|u^{*}(\tau(\tilde{t}))\right| \leq\left|\tau^{\prime}(\tilde{t})\right|$, which implies that according to Equation (12), $|u(\tilde{t})| \leq 1$ and then $K\left(u, x_{0}\right)=J$ in most cases, and that the integral given in Equation (17) can be solved easily. We will see in the following examples that in this case, the step two is key since if we choose properly $\tau$, then we will be able to find $y$ analytically and if we do not choose $\tau$ properly, we will not be able to find it. 
Finally, suppose that there is just one body and it moves in only one direction $x_{1}=x$ and we find a function $\tilde{x}$ that satisfies $\tilde{x}(\tilde{t}) \neq 0$ and $\tilde{x}\left(\tilde{t}_{0}\right)=x_{0}$. Then, the step one is satisfied. In addition, in this case the step two is unnecessary. Hence, the algorithm in this case becomes

1) Choose conveniently a function $\tilde{x}: J \rightarrow I R$ that satisfies $\tilde{x}^{\prime}(\tilde{t}) \neq 0$ and $\tilde{x}\left(\tilde{t}_{0}\right)=x_{0}$.

2) Find the function $u$ given in Equation (10) (or (13)).

3) Find a function $y$ that satisfies Equation (17).

As before, the statement "choose conveniently a function $\tilde{x}: J \rightarrow I R$ " refers to choose $\tilde{x}$ so that $|u(\tilde{t})| \leq 1$ and that the integral given in Equation (17) can be solved easily.

Finally, suppose that the universe is only composed of the $n$ bodies. Then, it would be impossible to tell if the bodies move according to $\overline{\bar{X}}$ and the time $t$ is "an horizontal line" or if the bodies move according to $\tilde{\overline{\bar{X}}}$ and the time curves according to Equation (17).

Hence, we can say that the master equation is a generalization of Newton's second law which includes the possibility that the time curves. We can also say that we do not know if Newton's second law is all right since we do not know if the time curves or does not.

Next we will see four examples. The first three examples will be one dimensional problems and then we will use this last algorithm.

\section{Examples}

In this section, we will solve the harmonic oscillator, the pendulum, the particle under the action of two elastic springs [2] and Kepler's problems. Note that all these problems have periodic solutions (at least for some values of the energy). We will use this formalism in order to approximate the periods. In order to do that we will need the following proposition.

Proposition 2: let $C$ be a curve with elliptical shape with semi-axes $a$ and $b$ and let $C_{1}$ and $C_{2}$ be two curves as shown in Figure 14. Then we have

$$
4 \sqrt{a^{2}+b^{2}}=l\left(C_{1}\right) \leq l(C) \leq l\left(C_{2}\right)=4(a+b)
$$

where $l(C), l\left(C_{1}\right)$ and $l\left(C_{2}\right)$ are the length of $C, C_{1}$ and $C_{2}$ respectively.

Suppose that $x(t)=\tilde{x}\left(x_{\gamma}(t)\right)$ is the solution of one of these problems with $C$ a closed curve with elliptical shape and semi-axes $a$ and $b$ and $\tilde{x}:[-a, a] \rightarrow l R$. If $\gamma$ turns indefinitely the solution holds for all $t$ and according to Figure 13 it is periodic. The period is given by the length of $C$ and then, according to this proposition, we have

$$
4 \sqrt{a^{2}+b^{2}} \leq \tau \leq 4(a+b)
$$

where $\tau$ is the period.

In other words, $\tau=\tau_{0} \pm \Delta \tau$ where

$$
\begin{gathered}
\tau_{0}=2\left(a+b+\sqrt{a^{2}+b^{2}}\right) \\
\Delta \tau=2\left(a+b-\sqrt{a^{2}+b^{2}}\right) .
\end{gathered}
$$

Hence, if we know $a$ and $b$, we can approximate the period of the solution using Equation (18) and the error of the approximation is given by Equation (19). If we call $\epsilon$ to the ratio between the semi-minor axis and the semi-major axis, according to Equations (18) and (19), the relative error is given by

$$
\frac{\Delta \tau}{\tau_{0}}=\frac{1+\epsilon-\sqrt{1+\epsilon^{2}}}{1+\epsilon+\sqrt{1+\epsilon^{2}}} .
$$

Since $0 \leq \epsilon \leq 1$, then it is easily proved that 




Figure 14. Graph of the curves $C, C_{1}$ and $C_{2}$.

$$
0 \leq \frac{\Delta \tau}{\tau_{0}} \leq \frac{2-\sqrt{2}}{2+\sqrt{2}}
$$

where $\frac{2-\sqrt{2}}{2+\sqrt{2}} \simeq 0.17$.

In addition, if $x=0$ is the equilibrium point, we can also calculate the amplitude of the motion by doing

$$
A=\max |x(t)|=\max _{\tilde{t} \in[-a, a]}|\tilde{x}(\tilde{t})| .
$$

Next we will see some examples and we will use these results in order to approximate the period of the solution. In all the examples we will consider just one body and we will denote by $X \equiv x_{1}$ and $Y \equiv x_{2}$. In the one dimensional examples we will use the second algorithm.

\subsection{Harmonic Oscillator}

The force and the potential in this case are given by

$$
\begin{aligned}
& F(X)=-k X \\
& V(X)=\frac{1}{2} k X^{2}
\end{aligned}
$$

where $k$ is the elastic constant.

Before proceeding, we will call

$$
\omega_{0}=\sqrt{\frac{k}{m}} .
$$

We propose as a solution the following one 


$$
\tilde{X}(\tilde{t})=v \tilde{t}
$$

where $v$ is given by

$$
v=\sqrt{\frac{2 E}{m}}
$$

with $E$ the mechanical energy.

We will choose

$$
\tilde{t}_{0}=x_{0}=\frac{X_{0}}{v} .
$$

Then, the solution satisfies $\tilde{X}^{\prime}(\tilde{t}) \neq 0$ (since we are not considering the trivial case $E=0$ ) and $\tilde{X}\left(\tilde{t}_{0}\right)=X_{0}$ and hence the first step of the algorithm is complete.

In order to find $u$, according to Equations (5), (23), (24), (25) and (26) we have

$$
\begin{gathered}
\tilde{T}(\tilde{t})=E \\
V(\tilde{X}(\tilde{t}))=E \omega_{0}^{2} \tilde{t}^{2} .
\end{gathered}
$$

In addition, according to Equations (11) and (25),

$$
\lambda=\frac{\dot{X}_{0}}{\tilde{X}^{\prime}\left(\tilde{t}_{0}\right)}=\frac{\dot{X}_{0}}{v} .
$$

Since $v$ is positive, then

$$
\operatorname{sgn}(\lambda)=\operatorname{sgn}\left(\dot{X}_{0}\right) .
$$

Finally, by Equations (13), (28), (29) and (30) the function $u$ is given by

$$
u(\tilde{t})=\operatorname{sgn}\left(\dot{X}_{0}\right) \sqrt{1-\omega_{0}^{2} \tilde{t}^{2}} .
$$

Then, the second step of the algorithm is complete.

In order to find $y$, according to Equation (31) we have

$$
\frac{1}{u^{2}(s)}-1=\frac{\omega_{0}^{2} s^{2}}{1-\omega_{0}^{2} s^{2}} \text {. }
$$

Then, by making the change of variable

$$
\mu=\omega_{0} \mathrm{~S}
$$

and choosing conveniently the value of $y_{0}$, Equation (17) becomes

$$
y(x)= \pm \frac{1}{\omega_{0}} \sqrt{1-\omega_{0}^{2} x^{2}} .
$$

Without being quite formal, it follows that the curve $C$ is given by

$$
y^{2}+x^{2}=\frac{1}{\omega_{0}^{2}} .
$$

Hence, the third step is complete.

Finally, $X(t)=\tilde{X}\left(x_{\gamma}(t)\right)$ is the solution where $\tilde{X}$ is given by Equation (25), $C$ is described by Equation (32), $P_{0}=\left(x_{0}, y\left(x_{0}\right)\right)$ with $x_{0}$ given in Equation (27) and the orientation is given according to the sign of the initial velocity. 
Since $C$ is a closed curve and we are considering that $\gamma$ turns indefinitely, we can graph $X$ versus $t$ as given in Figure 13. The solution is periodic and in this case, since the curve is a circumference of ratio $\frac{1}{\omega_{0}}$, we can calculate the period analytically and it is given by

$$
\tau=\frac{2 \pi}{\omega_{0}}
$$

In addition, according to Equations (22), (24), (25) and (26) the amplitude is given by

$$
A=\tilde{X}\left(\frac{1}{\omega_{0}}\right)=\frac{v}{\omega_{0}}=\sqrt{\frac{2 E}{k}} .
$$

We can find the period and the amplitude in the traditional way and we obtain the same results.

\subsection{The Pendulum}

We will call $\theta$ to the angle formed by the rope and the vertical and we will denote

$$
X=l \theta
$$

where $l$ is the length of the rope.

Then, the equation of motion can be written as Equation (1) where the force is given by

$$
F(X)=-m g \sin \left(\frac{X}{l}\right)
$$

and $g$ is the gravity.

We will choose conveniently the potential as follows

$$
V(X)=-2 m g l \cos ^{2}\left(\frac{X}{2 l}\right) .
$$

Before proceeding, using this equation and the fact that the kinetic energy is positive, note that

$$
E \geq-2 m g l \text {. }
$$

In addition, we will define conveniently

$$
\begin{gathered}
\omega_{0}=\sqrt{\frac{g}{l}} \\
\alpha=\sqrt{\frac{|E|}{2 m g l}} .
\end{gathered}
$$

We propose the solution of Equation (25) and we will take $\tilde{t}_{0}=x_{0}$ according to Equation (27) where in this case

$$
v=\sqrt{\frac{2 E}{m}+4 g l}=2 l \omega_{0} \sqrt{1+\operatorname{sgn}(E) \alpha^{2}} .
$$

By condition (36) we can see that $v$ is well defined.

As in the harmonic oscillator example, the solution satisfies $\tilde{X}^{\prime}(\tilde{t}) \neq 0$ and $\tilde{X}\left(\tilde{t}_{0}\right)=X_{0}$ which implies that the first step of the algorithm is complete.

In order to find $u$, according to Equations (5), (25), (35) and (39) we have

$$
\tilde{T}(\tilde{t})=E+2 m g l
$$




$$
V(\tilde{X}(\tilde{t}))=-2 m g l \cos ^{2}\left(\frac{v \tilde{t}}{2 l}\right)
$$

In addition, in a similar way we can prove Equation (32).

Finally, according to Equations (13), (32), (40) and (41) the function $u$ is given by

$$
u(\tilde{t})=\operatorname{sgn}\left(\dot{X}_{0}\right) \sqrt{\frac{E+2 m g l \cos ^{2}\left(\frac{v \tilde{t}}{2 l}\right)}{E+2 m g l}} .
$$

Then, the second step of the algorithm is complete.

In order to find $y$, according to Equation (42) we have

$$
\frac{1}{u^{2}(s)}-1=\frac{2 m g l \sin ^{2}\left(\frac{v s}{2 l}\right)}{E+2 m g l \cos ^{2}\left(\frac{v s}{2 l}\right)} .
$$

Then, by making the change of variable

$$
\mu=\sqrt{2 m g l} \cos \left(\frac{v s}{2 l}\right)
$$

and choosing conveniently the value of $y_{0}$, Equation (17) becomes

$$
y(x)= \pm \frac{2 l}{v}\left\{\begin{array}{l}
\operatorname{arccosh}\left(\frac{1}{\alpha} \cos \left(\frac{v x}{2 l}\right)\right) \text { if } E<0 \\
\ln \left(\cos \left(\frac{v x}{2 l}\right)\right) \text { if } E=0 \\
\operatorname{arcsinh}\left(\frac{1}{\alpha} \cos \left(\frac{v x}{2 l}\right)\right) \text { if } E>0 .
\end{array}\right.
$$

Without being quite formal, it follows that the curve $C$ is given by

$$
\left\{\begin{array}{l}
\alpha \cosh \left(\frac{v y}{2 l}\right)=\cos \left(\frac{v x}{2 l}\right) \text { if } E<0 \\
\mathrm{e}^{-\frac{v y}{2 l}}=\cos \left(\frac{v x}{2 l}\right) \text { if } E=0 \\
\alpha \sinh \left(\frac{v y}{2 l}\right)=\cos \left(\frac{v x}{2 l}\right) \text { if } E>0 .
\end{array}\right.
$$

According to Equation (39) we can also write $C$ as follows

$$
\left\{\begin{array}{l}
\alpha \cosh \left(\sqrt{1-\alpha^{2}} \omega_{0} y\right)=\cos \left(\sqrt{1-\alpha^{2}} \omega_{0} x\right) \text { if } E<0 \\
\mathrm{e}^{-\omega_{0} y}=\cos \left(\omega_{0} x\right) \text { if } E=0 \\
\alpha \sinh \left(\sqrt{1+\alpha^{2}} \omega_{0} y\right)=\cos \left(\sqrt{1+\alpha^{2}} \omega_{0} x\right) \text { if } E>0 .
\end{array}\right.
$$

Hence, the third step is complete.

Finally, $X(t)=\tilde{X}\left(x_{\gamma}(t)\right)$ is the solution where $\tilde{X}$ is given by Equation (25), $C$ is described by Equation (43), $P_{0}=\left(x_{0}, y\left(x_{0}\right)\right)$ with $x_{0}$ given in Equation (27) and the orientation is given according to the sign of the initial velocity. In addition, according to Equation (34) we have 


$$
\theta(t)=\frac{1}{l} \tilde{X}\left(x_{\gamma}(t)\right)=\tilde{\theta}\left(x_{\gamma}(t)\right)
$$

If $E<0$, then $C$ is a closed curve with elliptical shape. The semi-axes of the ellipse are given by

$$
\begin{gathered}
a=\frac{\arccos (\alpha)}{\sqrt{1-\alpha^{2}}} \frac{1}{\omega_{0}} \\
b=\frac{\operatorname{arccosh}\left(\frac{1}{\alpha}\right)}{\sqrt{1-\alpha^{2}}} \frac{1}{\omega_{0}} .
\end{gathered}
$$

It is easily proved that $b>a$ and hence the "ellipse is vertical".

Since $C$ is a closed curve and we are considering that $\gamma$ turns indefinitely, we can graph $X$ versus $t$ as in Figure 13. The solution is periodic and according to Equations (18), (44) and (45) its approximate period is given by

$$
\tau_{0}=\frac{\arccos (\alpha)+\operatorname{arccosh}\left(\frac{1}{\alpha}\right)+\sqrt{\arccos ^{2}(\alpha)+\operatorname{arccosh}^{2}\left(\frac{1}{\alpha}\right)}}{\sqrt{1-\alpha^{2}}} \frac{2}{\omega_{0}} .
$$

The relative error is given by Equation (20) where in this case

$$
\epsilon=\frac{a}{b}=\frac{\arccos \alpha}{\operatorname{arccosh}\left(\frac{1}{\alpha}\right)} .
$$

We will study the limit cases, i.e., when $E \rightarrow-2 m g l$ (see Equation (36)) and when $E \rightarrow 0$.

The first case corresponds to small oscillations and according to Equation (38) $\alpha \rightarrow 1$. Then, by Equation (47) it is easily proved that $\epsilon \rightarrow 1$ which implies that the curve is a circumference. Hence, as in the harmonic oscillator case, it is not necessary to approximate the period, since it can be calculated analytically. According to Equations (44) and (45) we have that $a=b \rightarrow \frac{1}{\omega_{0}}$ and then the ratio of the circumference takes the value of $\frac{1}{\omega_{0}}$. Hence, the period is given by Equation (33) where in this case $\omega_{0}$ is given by Equation (37) as was expected.

In the second case we have that $\alpha \rightarrow 0$ and then, Equations (46) and (47) becomes

$$
\begin{aligned}
\tau_{0} & \rightarrow \infty \\
\epsilon & \rightarrow 0 .
\end{aligned}
$$

Since $\epsilon \rightarrow 0$ we can see in Equation (20) that

$$
\frac{\Delta \tau}{\tau_{0}} \rightarrow 0
$$

Taking into account that in the previous case $\epsilon \rightarrow 1$ and then, according to Equations (20) and (21), the relative error is maximum, we can say that to higher energy, better approximation in the period. However, by Equations (19), (44) and (45) it is easily proved that the error depending on $\alpha$ is a decreasing function and it tends to infinity when $\alpha \rightarrow 0$.

Finally, we can also find the amplitude and according to Equations (22), (25), (39) and (44) it is given by

$$
A=\tilde{X}(a)=v a=2 l \arccos (\alpha) .
$$

We can find the amplitude in the traditional way and see that we arrive to the same result. 
If $E=0$ the graph of the curve is shown in Figure 15 where $a$ is given by

$$
a=\frac{\pi}{2 \omega_{0}} .
$$

If we graph $X$ versus $t$ as in Figure 12 and we assume that the initial velocity is positive we can see that the solution is increasing and since the curve has vertical asymptotes in $\pm a$, according to Equations (25) and (39) it tends to

$$
\tilde{X}(a)=l \pi
$$

However, it never reaches that value.

If $E>0$ the graph of the curve is shown in Figure 16 where $a$ and $b$ are given by

$$
\begin{gathered}
a=\frac{\pi}{2} \frac{1}{\sqrt{1-\alpha^{2}}} \frac{1}{\omega_{0}} \\
b=\frac{\operatorname{arcsinh}\left(\frac{1}{\alpha}\right)}{\sqrt{1-\alpha^{2}}} \frac{1}{\omega_{0}} .
\end{gathered}
$$

In this case, if we graph $X$ versus $t$ as in Figure 12 and we assume that the initial velocity is positive, we can see that $X$ increases indefinitely and hence according to Equation (34) $\theta$ increases too. This means that the pendulum

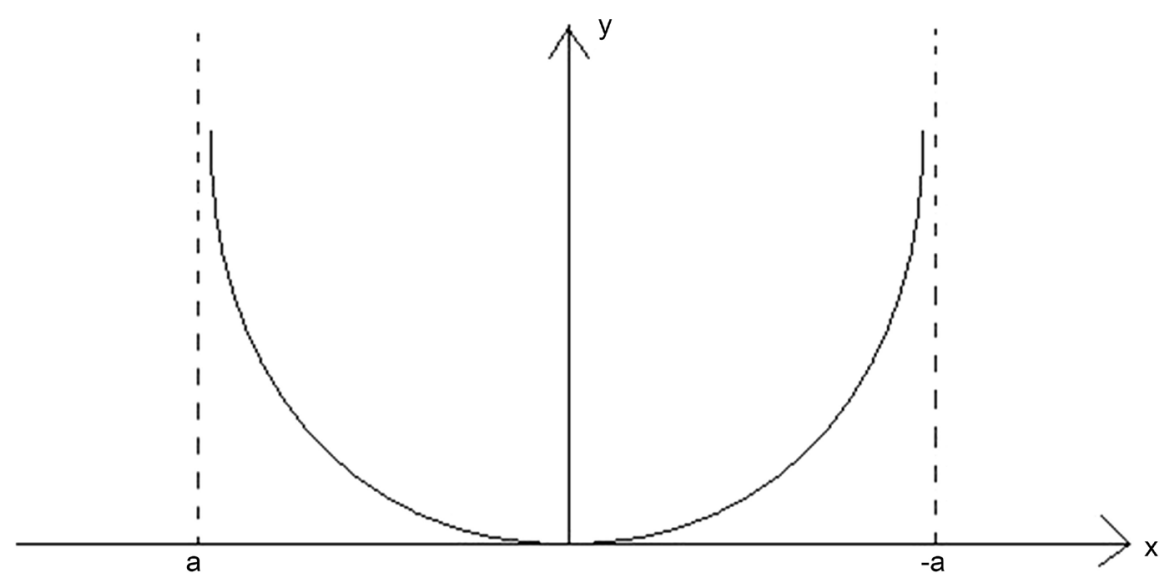

Figure 15. Graph of the curve $C$ for $E=0$.

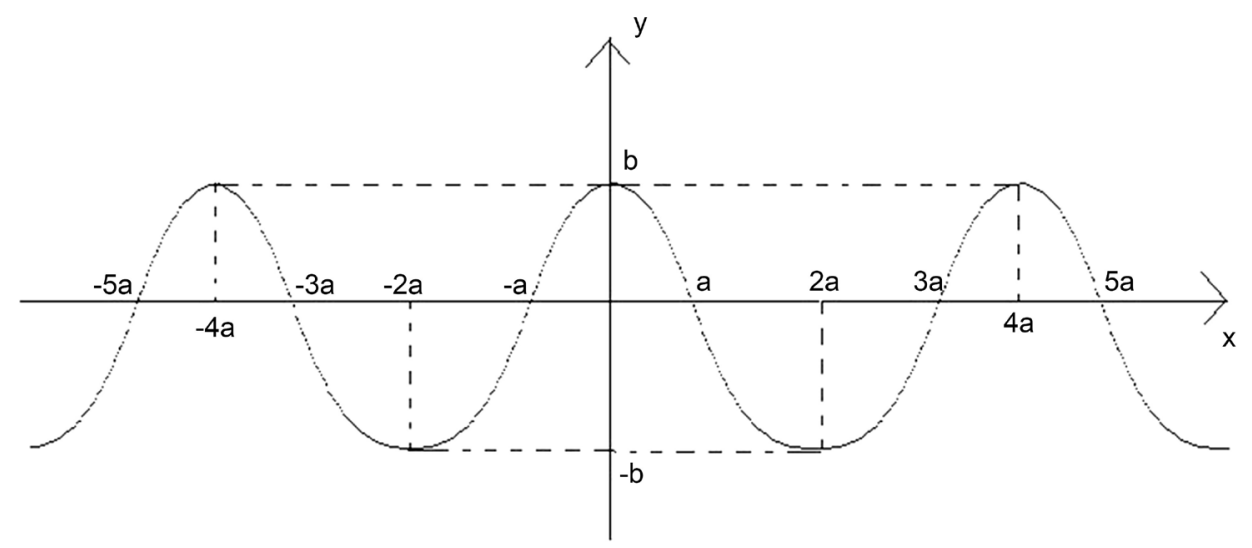

Figure 16. Graph of the curve $C$ for $E>0$. 
describes a rotating circular motion. The time $\tau$ that takes to the pendulum to circle is given by the length of the curve from $x_{0}$ to $x_{0}+\frac{2 \pi l}{v}$, since according to Equations (25), (27) and (34)

$$
\tilde{\theta}\left(x_{0}+\frac{2 \pi l}{v}\right)=\frac{1}{l} \tilde{X}\left(x_{0}+\frac{2 \pi l}{v}\right)=\frac{1}{l}\left(X_{0}+2 \pi l\right)=\theta_{0}+2 \pi .
$$

As in Proposition 2, we can obtain lower bounds and upper bounds for this length and approximate $\tau$ in the same way as before. For example if $X_{0}=0$, taking into account that according to Equations (27), (39) and (48)

$$
\begin{gathered}
x_{0}=0 \\
x_{0}+\frac{2 \pi l}{v}=2 a
\end{gathered}
$$

it is easily proved that $\tau=\tau_{0} \pm \Delta \tau$ where

$$
\begin{gathered}
\tau_{0}=\left(a+b+\sqrt{a^{2}+b^{2}}\right) \\
\Delta \tau=\left(a+b-\sqrt{a^{2}+b^{2}}\right)
\end{gathered}
$$

with $a$ and $b$ given by Equations (48) and (49).

\subsection{Particle under the Action of Two Elastic Springs}

The force and the potential in this case, considering small oscillations, are given by [2]

$$
\begin{aligned}
& F(X)=-\frac{k}{l_{0}^{2}} X^{3} \\
& V(X)=\frac{1}{4} \frac{k}{l_{0}^{2}} X^{4}
\end{aligned}
$$

where $k$ is the elastic constant and $l_{0}$ is the natural length of the springs.

We propose as a solution

$$
\tilde{X}(\tilde{t})=A \sinh (\omega \tilde{t})
$$

where

$$
\begin{gathered}
A=\sqrt[4]{\frac{4 l_{0}^{2} E}{k}}=\sqrt[4]{\frac{4 E}{k l_{0}^{2}}} l_{0} \\
\omega=\sqrt[4]{\frac{k E}{l_{0}^{2} m^{2}}}=\sqrt[4]{\frac{E}{k l_{0}^{2}}} \omega_{0}
\end{gathered}
$$

with $\omega_{0}$ given in Equation (24) and $E$ the mechanical energy.

We will choose

$$
\tilde{t}_{0}=x_{0}=\frac{1}{\omega} \operatorname{arcsinh}\left(\frac{X_{0}}{A}\right) .
$$

Then, the solution satisfies $\tilde{X}^{\prime}(\tilde{t}) \neq 0$ (since we are not considering the trivial case $E=0$ ), $\quad \tilde{X}\left(\tilde{t}_{0}\right)=X_{0}$ and hence the first step of the algorithm is complete.

In order to find $u$, according to Equations (5), (50), (51), (52) and (53) we have

$$
\tilde{T}(\tilde{t})=E \cosh ^{2}(\omega \tilde{t})
$$




$$
V(\tilde{X}(\tilde{t}))=E \sinh ^{4}(\omega \tilde{t}) .
$$

In addition, according to Equations (11) and (51),

$$
\lambda=\frac{\dot{X}_{0}}{\tilde{X}^{\prime}\left(\tilde{t}_{0}\right)}=\frac{\dot{X}_{0}}{A \omega \cosh \left(\omega \tilde{t}_{0}\right)} .
$$

Since $A, \omega$ and $\cosh \left(\omega \tilde{t}_{0}\right)$ are positive, then Equation (30) holds.

Finally, according to Equations (13), (30), (55) and (56) the function $u$ is given by

$$
u(\tilde{t})=\operatorname{sgn}\left(\dot{X}_{0}\right) \sqrt{\frac{1-\sinh ^{4}(\omega \tilde{t})}{\cosh ^{2}(\omega \tilde{t})}} .
$$

Then, the second step of the algorithm is complete.

In order to find $y$, according to Equation (57) we have

$$
\frac{1}{u^{2}(s)}-1=\frac{\cosh ^{2}(\omega s) \sinh ^{2}(\omega s)}{1-\sinh ^{4}(\omega s)} .
$$

Then, by making the change of variable

$$
\mu=\sinh ^{2}(\omega s)
$$

and choosing conveniently the value of $y_{0}$, Equation (17) becomes

$$
y(x)= \pm \frac{1}{2 \omega} \arccos \left(\sinh ^{2}(\omega x)\right) .
$$

Without being quite formal, it follows that the curve $C$ is given by

$$
\cos (2 \omega y)=\sinh ^{2}(\omega x) .
$$

Using well known properties of the trigonometric and the hyperbolic functions we can write this equation as follows

$$
\cos (\omega y)=\frac{1}{\sqrt{2}} \cosh (\omega x)
$$

Hence, the third step is complete.

Finally, $X(t)=\tilde{X}\left(x_{\gamma}(t)\right)$ is the solution where $\tilde{X}$ is given by Equation (51), $C$ is described by Equation (58), $P_{0}=\left(x_{0}, y\left(x_{0}\right)\right)$ with $x_{0}$ given in Equation (54) and the orientation is given according to the sign of the initial velocity.

It is proved that $C$ is a closed curve with elliptical shape. The semi-axes $a$ and $b$ are given by

$$
\begin{aligned}
& a=\operatorname{arccosh}(\sqrt{2}) \frac{1}{\omega} \simeq \frac{0.88}{\omega} \\
& b=\arccos \left(\frac{1}{\sqrt{2}}\right) \frac{1}{\omega} \simeq \frac{0.79}{\omega} .
\end{aligned}
$$

In this case $a>b$ and hence the "ellipse is horizontal".

Since $C$ is a closed curve and we are considering that $\gamma$ turns indefinitely, we can graph $X$ versus $t$ as in Figure 13. The solution is periodic and according to Equations (18), (58) and (59) its approximate period is given by

$$
\tau_{0} \simeq \frac{5.69}{\omega} .
$$


The relative error, according to Equation (20) and taking into account that

$$
\epsilon=\frac{b}{a} \simeq 0.90
$$

is given by

$$
\frac{\Delta \tau}{\tau_{0}} \simeq 0.17
$$

Note that if $E \rightarrow 0$ (which implies small oscillations), then $\omega \rightarrow 0$ (see Equation (53)) and hence $\tau_{0} \rightarrow \infty$. On the other hand, if $E \rightarrow \infty$, then $\omega \rightarrow \infty$ and hence $\tau_{0} \rightarrow 0$.

By means of the procedure indicated before, it is easily proved that $A$ is the amplitude.

\subsection{Kepler's Problem}

The force and the potential in this case are given by

$$
\begin{gathered}
\bar{F}(\bar{r})=-\alpha \frac{\bar{r}}{r^{3}} \\
V(\bar{r})=-\frac{\alpha}{r}
\end{gathered}
$$

where $\bar{r}=(x, y), \quad r=\|\bar{r}\|$ and $\alpha$ is a constant.

We will assume that the initial conditions are given by

$$
\left\{\begin{array}{l}
\bar{r}(0)=\left(r_{0}, 0\right) \\
\dot{\bar{r}}(0)=\left(0, v_{0}\right)
\end{array}\right.
$$

where $r_{0}$ and $v_{0}$ are positive.

In addition, we will choose $\tilde{t}_{0}=x_{0}=0$.

Then, since $\left\{\left(\bar{T}_{y},-\bar{T}_{x}\right)\right\}$ is a base of the $\langle\tilde{\bar{T}}\rangle$ orthogonal subspace (see the appendix of [1]) and $T_{x}(0)=0$ (because $\dot{x}(0)=0$ ), the trajectory equation becomes

$$
\left\{\begin{array}{l}
\tilde{W}_{x}(\tilde{t}) \tilde{T}_{y}(\tilde{t})-\left(\tilde{W}_{y}(\tilde{t})+T(0)\right) \tilde{T}_{x}(\tilde{t})=0 \\
\left(r_{0}, 0\right)=\tilde{\bar{r}}(0) \\
\left(0, v_{0}\right) / / \tilde{r}^{\prime}(0)
\end{array}\right.
$$

where according to Equations (4), (6) and (62)

$$
\begin{gathered}
T(0)=T_{y}(0)=\frac{1}{2} m v_{0}^{2} \\
\tilde{W}_{x}(\tilde{t})=-\alpha \int_{0}^{\tilde{t}} \frac{\tilde{x}(s) \tilde{x}^{\prime}(s)}{\tilde{r}^{3}(s)} \mathrm{d} s \\
\tilde{W}_{y}(\tilde{t})=-\alpha \int_{0}^{\tilde{t}} \frac{\tilde{y}(s) \tilde{y}^{\prime}(s)}{\tilde{r}^{3}(s)} \mathrm{d} s .
\end{gathered}
$$

We propose the following solution

$$
\left\{\begin{array}{l}
\tilde{x}(\tilde{t})=A_{x} \cos (\omega \tilde{t})+B_{x} \\
\tilde{y}(\tilde{t})=A_{y} \sin (\omega \tilde{t})
\end{array}\right.
$$

where $A_{x}, B_{x}$ are real constants and $A_{y}, w$ can be complex. 
We have

$$
\begin{aligned}
\tilde{r}^{2}(\tilde{t}) & =\tilde{x}^{2}(\tilde{t})+\tilde{y}^{2}(\tilde{t})=A_{x}^{2} \cos ^{2}(\omega \tilde{t})+2 A_{x} B_{x} \cos (\omega \tilde{t})+B_{x}^{2}+A_{y}^{2} \sin ^{2}(\omega \tilde{t}) \\
& =\left(A_{x}^{2}-A_{y}^{2}\right) \cos ^{2}(\omega \tilde{t})+2 A_{x} B_{x} \cos (\omega \tilde{t})+A_{y}^{2}+B_{x}^{2} .
\end{aligned}
$$

Let

$$
\mu(\tilde{t})=\cos (\omega \tilde{t})
$$

then we arrive to

$$
\tilde{r}^{2}(\tilde{t})=\left(A_{x}^{2}-A_{y}^{2}\right) \mu^{2}(\tilde{t})+2 A_{x} B_{x} \mu(\tilde{t})+A_{y}^{2}+B_{x}^{2} .
$$

In order to solve the integrals given in Equation (67) and (68), we will ask that this expression is a perfect square. It happens if and only if

$$
B_{x}^{2}=A_{x}^{2}-A_{y}^{2}
$$

where we assume that $A_{x} \geq A_{y}$, provided $A_{y}$ is real.

In this case we obtain

$$
\tilde{r}^{2}(\tilde{t})=\left(B_{x} \mu(\tilde{t})+A_{x}\right)^{2}
$$

We will assume that

$$
B_{x} \mu(\tilde{t})+A_{x} \geq 0 \quad \forall \tilde{t} \in J
$$

and hence we finally arrive to

$$
\tilde{r}(\tilde{t})=B_{x} \mu(\tilde{t})+A_{x} .
$$

On the other hand, according to Equation (69) we have

$$
\left\{\begin{array}{l}
\tilde{x}^{\prime}(\tilde{t})=-A_{x} \omega \sin (\omega \tilde{t}) \\
\tilde{y}^{\prime}(\tilde{t})=A_{y} \omega \cos (\omega \tilde{t})
\end{array} .\right.
$$

Then, by Equation (69) it follows that

$$
\begin{gathered}
\tilde{x}(\tilde{t}) \tilde{x}^{\prime}(\tilde{t})=-\omega\left(A_{x}^{2} \sin (\omega \tilde{t}) \cos (\omega \tilde{t})+A_{x} B_{x} \sin (\omega \tilde{t})\right) \\
\tilde{y}(\tilde{t}) \tilde{y}^{\prime}(\tilde{t})=\omega A_{y}^{2} \sin (\omega \tilde{t}) \cos (\omega \tilde{t}) .
\end{gathered}
$$

We can write these expressions using Equation (70) as follows

$$
\begin{gathered}
\tilde{x}(\tilde{t}) \tilde{x}^{\prime}(\tilde{t})=A_{x}^{2} \mu(\tilde{t}) \mu^{\prime}(\tilde{t})+A_{x} B_{x} \mu^{\prime}(\tilde{t}) \\
\tilde{y}(\tilde{t}) \tilde{y}^{\prime}(\tilde{t})=-A_{y}^{2} \mu(\tilde{t}) \mu^{\prime}(\tilde{t}) .
\end{gathered}
$$

Hence, by Equations (73), (75) and (76), we finally obtain that Equations (67) and (68) becomes

$$
\begin{gathered}
\tilde{W}_{x}(\tilde{t})=-\alpha \int_{0}^{\tilde{t}} \frac{A_{x}^{2} \mu(s) \mu^{\prime}(s)+A_{x} B_{x} \mu^{\prime}(s)}{\left(B_{x} \mu(s)+A_{x}\right)^{3}} \mathrm{~d} s \\
\tilde{W}_{y}(\tilde{t})=\alpha \int_{0}^{\tilde{t}} \frac{A_{y}^{2} \mu(s) \mu^{\prime}(s)}{\left(B_{x} \mu(s)+A_{x}\right)^{3}} \mathrm{~d} s .
\end{gathered}
$$

Let 


$$
\begin{aligned}
& K_{1}(\mu)=\int \frac{\mu}{\left(B_{x} \mu+A_{x}\right)^{3}} \mathrm{~d} \mu=\frac{2 B_{x} \mu+A_{x}}{2 B_{x}^{2}\left(B_{x} \mu+A_{x}\right)^{2}} \\
& K_{2}(\mu)=\int \frac{1}{\left(B_{x} \mu+A_{x}\right)^{3}} \mathrm{~d} \mu=\frac{1}{2 B_{x}\left(B_{x} \mu+A_{x}\right)^{2}}
\end{aligned}
$$

where we made the change of variables $v=B_{x} \mu+A_{x}$ for solving the integrals.

We can write Equations (77) and (78) as follows

$$
\begin{gathered}
\tilde{W}_{x}(\tilde{t})=\alpha A_{x}^{2}\left(K_{1}(\mu(\tilde{t}))-K_{1}(1)\right)+\alpha A_{x} B_{x}\left(K_{2}(\mu(\tilde{t}))-K_{2}(1)\right) \\
\tilde{W}_{y}(\tilde{t})=\alpha A_{y}^{2}\left(K_{1}(1)-K_{1}(\mu(\tilde{t}))\right) .
\end{gathered}
$$

On the other hand, by Equations (5) and (74) we have

$$
\begin{aligned}
& \tilde{T}_{x}(\tilde{t})=\frac{1}{2} m A_{x}^{2} \omega^{2} \sin ^{2}(\omega \tilde{t}) \\
& \tilde{T}_{y}(\tilde{t})=\frac{1}{2} m A_{y}^{2} \omega^{2} \cos ^{2}(\omega \tilde{t}) .
\end{aligned}
$$

Using Equation (70) we arrive to

$$
\begin{gathered}
\tilde{T}_{x}(\tilde{t})=\frac{1}{2} m A_{x}^{2} \omega^{2}\left(1-\mu^{2}(\tilde{t})\right) \\
\tilde{T}_{y}(\tilde{t})=\frac{1}{2} m A_{y}^{2} \omega^{2} \mu^{2}(\tilde{t}) .
\end{gathered}
$$

Finally, by Equations (81), (82), (83) and (84) we can turn Equation (65) into an algebraic equation as follows

$$
\begin{aligned}
& {\left[\alpha A_{x}^{2}\left(K_{1}(\mu)-K_{1}(1)\right)+\alpha A_{x} B_{x}\left(K_{2}(\mu)-K_{2}(1)\right)\right] A_{y}^{2} \mu^{2}} \\
& =\left[\alpha A_{y}^{2}\left(K_{1}(1)-K_{1}(\mu)\right)+T(0)\right] A_{x}^{2}\left(1-\mu^{2}\right) .
\end{aligned}
$$

This equation can be written as

$$
\begin{aligned}
& \alpha A_{x} A_{y}^{2} B_{x} \mu^{2} K_{2}(\mu)+\alpha A_{x}^{2} A_{y}^{2} K_{1}(\mu) \\
& =\left(\alpha A_{x} A_{y}^{2} B_{x} K_{2}(1)-A_{x}^{2} T(0)\right) \mu^{2}+\left(\alpha A_{x}^{2} A_{y}^{2} K_{1}(1)+A_{x}^{2} T(0)\right) .
\end{aligned}
$$

In order to satisfy this equality for all $\mu$, we will ask

$$
A_{x}^{2} T(0)=\alpha A_{x} A_{y}^{2} B_{x} K_{2}(1) .
$$

Then, Equation (85) becomes

$$
B_{x} \mu^{2} K_{2}(\mu)+A_{x} K_{1}(\mu)=A_{x} K_{1}(1)+B_{x} K_{2}(1) .
$$

This equation depends only on $A_{x}$ and $B_{x}$ and using Equations (79) and (80) it is easily proved that it is satisfied for all $\mu$.

In order to satisfy $\left(r_{0}, 0\right)=\tilde{\bar{r}}(0)$, according to Equation (69) we have to ask

$$
A_{x}+B_{x}=r_{0} .
$$

By Equation (74), the other initial condition $\left(0, v_{0}\right) / / \tilde{\bar{r}}^{\prime}(0)$ is satisfied by taking

$$
\lambda=\frac{v_{0}}{w A_{y}}
$$


where $\lambda$ is given in Equation (11).

Finally, the trajectory equation is satisfied if and only if Equations (71), (86) and (87) hold, provided that $B_{x}$ and $A_{x}$ are real and that condition (72) is satisfied. This is a system of three equations where $A_{x}, A_{y}$ and $B_{x}$ are the unknowns. Using Equations (66) and (80) it is proved that the solution is given by

$$
\begin{aligned}
A_{x} & =-\frac{\alpha}{2 E} \\
A_{y}^{2} & =-\frac{l^{2}}{2 m E} \\
B_{x} & =r_{0}+\frac{\alpha}{2 E}
\end{aligned}
$$

where $l$ is the angular momentum (which is known that is constant).

However, this solution holds only if $A_{x}^{2} \geq A_{y}^{2}$. Using Equations (89) and (90) this condition is equivalent to

$$
E \geq-\frac{m \alpha^{2}}{2 l^{2}}
$$

By this condition, we can obtain the minimum value of $v_{0}$ and then it is easily proved that the minimum value of $E$ is given by

$$
E_{0}=-\frac{\alpha}{2 r_{0}} .
$$

If $A_{x}^{2}<A_{y}^{2}$ we can see in Equation (71) that $B_{x}$ is complex but according to Equation (91) $B_{x}$ is real which is a contradiction. Then the system of equations has not solution in the case $A_{x}^{2}<A_{y}^{2}$ and hence the solution given in Equation (69) does not work.

Next we will choose $\omega$ in order to satisfy condition (72). Provided that $E \geq E_{0}$ there are two cases, $E<0$ or $E>0$ (we will not consider the case $E=0$ ).

In the first case, according to Equations (89) and (90), $A_{x}$ is positive and $A_{y}$ is real. In addition, using Equation (91) and the fact that $E \geq E_{0}$ with $E_{0}$ given in Equation (92) it is proved that $B_{x}$ is negative. In this case we will choose $\omega=\omega_{0}$ with $\omega_{0} \in l R$. Then, according to Equation (70) we have

$$
\mu(\tilde{t})=\cos (\omega \tilde{t})=\cos \left(\omega_{0} \tilde{t}\right) \geq-1 .
$$

Using this result, and taking into account that $A_{x}$ is positive and $B_{x}$ is negative it is proved that condition (72) is satisfied.

In the second case, according to Equations (89), (90) and (91), $A_{x}$ is negative, $A_{y}$ is a pure imaginary complex number and $B_{x}$ is positive. In this case we will choose $\omega=i \omega_{0}$ with $i$ the imaginary unit and $\omega_{0} \in I R$. Then, according to Equations (70) and (71) we have

$$
\begin{gathered}
\mu(\tilde{t})=\cos (\omega \tilde{t})=\cos \left(i \omega_{0} \tilde{t}\right)=\cosh \left(\omega_{0} \tilde{t}\right) \geq 1 \\
B_{x}=\sqrt{A_{x}^{2}-A_{y}^{2}}=\sqrt{A_{x}^{2}+\left|A_{y}\right|^{2}} \geq\left|A_{x}\right|=-A_{x} .
\end{gathered}
$$

Using these results it is proved that condition (72) is satisfied.

Finally, using that

$$
\begin{array}{r}
\cos (i x)=\cosh (x) \\
-i \sin (i x)=\sinh (x)
\end{array}
$$

the solution of the trajectory equation is given by

$$
\tilde{r}(\tilde{t})=\left\{\begin{array}{l}
\left(A_{x} \cos \left(\omega_{0} \tilde{t}\right)+B_{x},\left|A_{y}\right| \sin \left(\omega_{0} \tilde{t}\right)\right) \text { if } E_{0} \leq E<0 \\
\left(A_{x} \cosh \left(\omega_{0} \tilde{t}\right)+B_{x},\left|A_{y}\right| \sinh \left(\omega_{0} \tilde{t}\right)\right) \text { if } E>0
\end{array}\right.
$$


where $A_{x},\left|A_{y}\right|$ and $B_{x}$ are given in Equations (89), (90) and (91) and $E_{0}$ is given in Equation (92).

It is worthwhile to point out that we choose $A_{y}$ in the following way

$$
A_{y}=\left\{\begin{array}{l}
\left|A_{y}\right| \text { if } E_{0} \leq E<0 \\
i\left|A_{y}\right| \text { if } E>0
\end{array} .\right.
$$

If $E<0$, according to Equation (93), $\tilde{r}$ is the parametrization of an ellipse as was expected. The semimajor and semi-minor axis are given by $A_{x}$ and $A_{y}$ respectively and the center is given by $\left(B_{x}, 0\right)$. According to Equation (71), the focus of the ellipse is in the origin as Kepler's first law says. We can obtain the semi-axes and the center in the traditional way and check that we arrive to Equations (89), (90) and (91). In addition, we can calculate the minimum value of the energy under the hypothesis we have a bound motion and check that we obtain Equation (92).

We can see in Equation (93) that $\tilde{r}^{\prime}(\tilde{t}) \neq 0$ and that

$$
\tilde{x}_{i}^{\prime}(\tilde{t})=0 \Rightarrow \tilde{x}_{i}^{\prime \prime}(\tilde{t}) \neq 0 .
$$

Hence, conditions (8) and (9) are satisfied and then the first step of the algorithm is complete.

We will just choose $\tau(\tilde{t})=\tilde{t}$ to complete the second step.

In order to find $u$, on the one hand, according to Equations (63) and (73) we have

$$
E-V(\tilde{\bar{r}}(\tilde{t}))=E+\frac{\alpha}{B_{x} \mu(\tilde{t})+A_{x}}=\frac{E B_{x} \mu(\tilde{t})+E A_{x}+\alpha}{B_{x} \mu(\tilde{t})+A_{x}} .
$$

By Equation (89) this expression turns out to be

$$
E-V(\tilde{\tilde{r}}(\tilde{t}))=E \frac{B_{x} \mu(\tilde{t})-A_{x}}{B_{x} \mu(\tilde{t})+A_{x}} .
$$

On the other hand, according to Equations (83) and (84) we obtain

$$
\tilde{T}(\tilde{t})=\tilde{T}_{x}(\tilde{t})+\tilde{T}_{x}(\tilde{t})=\frac{1}{2} m \omega^{2}\left[A_{x}^{2}+\left(A_{y}^{2}-A_{x}^{2}\right) \mu^{2}(\tilde{t})\right] .
$$

According to Equation (71) we arrive to

$$
\tilde{T}(\tilde{t})=-\frac{1}{2} m \omega^{2}\left(B_{x} \mu(\tilde{t})+A_{x}\right)\left(B_{x} \mu(\tilde{t})-A_{x}\right) .
$$

Finally, according to Equations (13), (95) and (96) we have

$$
u^{2}(\tilde{t})=-\frac{2 E}{m \omega^{2}} \frac{1}{\left(B_{x} \mu(\tilde{t})+A_{x}\right)^{2}} .
$$

Using Equations (89) and (91) we can write this expression as follows

$$
u^{2}(\tilde{t})=-\frac{8 E^{3}}{m \alpha^{2} \omega^{2}} \frac{A_{x}^{2}}{\left(B_{x} \mu(\tilde{t})+A_{x}\right)^{2}} .
$$

We will choose $\omega$ in order to satisfy

$$
-\frac{8 E^{3}}{m \alpha^{2} \omega^{2}}=1 .
$$

Note that $E<0$ implies that $\omega$ is real and $E>0$ implies that $\omega$ is complex. Then this is consistent with the results obtained above. In addition, we will consider $\omega_{0}>0$ in both cases. Then we have

$$
\omega= \begin{cases}\omega_{0} & \text { if } E_{0} \leq E<0 \\ i \omega_{0} & \text { if } E>0\end{cases}
$$


where $\omega_{0}$ is given by

$$
\omega_{0}=\sqrt{\frac{8|E|^{3}}{m \alpha^{2}}} .
$$

Finally, Equation (97) turns out to be

$$
u^{2}(\tilde{t})=\frac{A_{x}^{2}}{\left(B_{x} \mu(\tilde{t})+A_{x}\right)^{2}} .
$$

On the other hand, using Equation (88) and the fact that $v_{0}$ is positive we arrive to

$$
\operatorname{sgn}(u)=\operatorname{sgn}(\lambda)=\operatorname{sgn}\left(A_{y} \omega_{0}\right) .
$$

In addition, by Equations (94) and (98) it is easily proved that

$$
\operatorname{sgn}\left(A_{y} \omega_{0}\right)=-\operatorname{sgn}(E)
$$

which implies

$$
\operatorname{sgn}(u)=-\operatorname{sgn}(E)
$$

Finally, by Equations (100) and (101) and using condition (72) we obtain

$$
u(\tilde{t})=-\operatorname{sgn}(E) \frac{\left|A_{x}\right|}{\left(B_{x} \mu(\tilde{t})+A_{x}\right)} .
$$

However, according to Equation (89)

$$
\operatorname{sgn}(E)=-\operatorname{sgn}\left(A_{x}\right)
$$

and then we just can write

$$
u(\tilde{t})=\frac{A_{x}}{\left(B_{x} \mu(\tilde{t})+A_{x}\right)} .
$$

Using Equations (70), (89) and (91) this equation can be written as follows

$$
u(\tilde{t})=\frac{1}{1-\kappa \cos (\omega \tilde{t})}
$$

where $\kappa$ is given by

$$
\kappa=-\frac{B_{x}}{A_{x}}=1-\frac{E}{E_{0}}
$$

and $E_{0}$ is given in Equation (92).

Note that since $E \geq E_{0}$ and $E_{0}<0$ then $\kappa$ is positive.

Finally we complete the third step.

In this case we will not find the function $y$ since it is not easy to find a function $\tau$ in order to solve the integral of Equation (17). However, in this case we can solve the temporal equation implicitly. According to Equations (2) and (102) and taking into account that $t_{0}=\tilde{t}_{0}=0$ this equation becomes

$$
\left\{\begin{array}{l}
\dot{\tilde{t}}(t)=\frac{1}{1-\kappa \cos (\omega \tilde{t})} \\
\tilde{t}(0)=0 .
\end{array}\right.
$$


It is easily proved that the solution is given by

$$
t=\tilde{t}(t)-\frac{\kappa}{\omega} \sin (\omega \tilde{t}(t)) .
$$

By Equation (98) we can write the solution depending the case as follows

$$
t=\left\{\begin{array}{l}
\tilde{t}(t)-\frac{\kappa}{\omega_{0}} \sin \left(\omega_{0} \tilde{t}(t)\right) \text { if } E<0 \\
\tilde{t}(t)-\frac{\kappa}{\omega_{0}} \sinh \left(\omega_{0} \tilde{t}(t)\right) \text { if } E>0
\end{array}\right.
$$

where $\omega_{0}$ is given in Equation (99).

Finally, $\bar{r}(t)=\tilde{r}(\tilde{t}(t))$ is the solution of Kepler's problem (with the initial conditions given in Equation (64)) where $\tilde{r}$ is given in Equation (93) and $\tilde{t}(t)$ is given by Equation (104).

Finally, we will obtain Kepler's third law which says that the square of the orbital period is directly proportional to the cube of the semi-major axis.

In Equation (93) we can see that if $E<0$, then $\tilde{r}$ is a periodic function whose period is given by $\frac{2 \pi}{\omega_{0}}$. Then, according to Equation (104), the time it takes to “the planet” to make a full turn is given by

$$
\tau=\frac{2 \pi}{\omega_{0}}-\frac{\kappa}{\omega_{0}} \sin \left(\omega_{0} \frac{2 \pi}{\omega_{0}}\right)=\frac{2 \pi}{\omega_{0}} .
$$

Using Equations (89) and (99) we finally arrive to

$$
\tau^{2}=4 \pi^{2} \frac{m}{\alpha} A_{x}^{3}
$$

and then we proved Kepler's third law.

\section{Conclusion}

We used the arc length function and its inverse in order to introduce a new way to graph functions by curving the axes as shown in Figure 10 and Figure 12. Using this, we found a more conveniently method to solve the temporal equation and then we changed the fourth step of the algorithm given in the first part in order to graph each component of the solution of Equation (1) as given in the mentioned figures. Following this algorithm, we solved the harmonic oscillator, the pendulum, the particle under the action of two elastic springs and Kepler's problems. We were able to solve the pendulum and the particle under the action of two elastic springs problems without using elliptical integrals and to see the periods of both problems as the length of a curve given in Equations (43) and (58) respectively. Then, using this fact, we approximate the periods of both problems in Equations (46) and (61) with a relative error less than 0.17. Finally, in Kepler's problem, we solved the trajectory equation and we proved that the solution describes an ellipse with focus in the origin (for some values of the energy). We could obtain the semi-major and semi-minor axes, the center of the ellipse and the orbital period by using this formalism.

\section{References}

[1] Petrovich, F. (2016) A New Formulation of Classical Mechanics-Part 1. Journal of Applied Mathematics and Physics, 4, 412-431. http://dx.doi.org/10.4236/jamp.2016.42048

[2] Goldstein, H. (1950) Classical Mechanics. Eddison-Wesley, Reading, MA.

[3] http://www.sc.ehu.es/sbweb/fisica_//oscilaciones/no_lineales/nolineal/nolineal_1.html 